\title{
Toxoplasma GRA15 limits parasite growth in IFNy-activated fibroblasts through TRAF ubiquitin ligases
}

Debanjan Mukhopadhyay ${ }^{1}$, Lamba Omar Sangaré ${ }^{1}$, Laurence Braun², Mohamed-Ali Hakimi², Jeroen P.J.

Saeij $^{1^{*}}$

1 Department of Pathology, Microbiology and Immunology, University of California Davis, Davis, California, United States of America

2 Institute for Advanced Biosciences, Team Host-Pathogen Interactions and Immunity to Infection, INSERM U 1209, CNRS UMR 5309, Université Grenoble Alpes, Grenoble, France

Running title: GRA15 aids IFNy-induced parasite killing

Key words: GRA15/IFNy/p62/Toxoplasma/TRAF6

\section{Abstract}

The protozoan parasite Toxoplasma gondii lives inside a vacuole in the host cytoplasm where it is protected from host cytoplasmic innate immune responses. However, IFNy-dependent cell-autonomous immunity can destroy the vacuole and the parasite inside. Toxoplasma strain differences in susceptibility to human IFNy exist but the Toxoplasma effector(s) that determine these differences are unknown. We show that in human primary fibroblasts, the polymorphic Toxoplasma secreted effector GRA15 mediates the recruitment of ubiquitin ligases, including TRAF2 and TRAF6, to the vacuole membrane, which enhances recruitment of ubiquitin receptors (p62/NDP52) and ubiquitin-like molecules (LC3B, GABARAP). This ultimately leads to lysosomal degradation of the vacuole. In murine fibroblasts, GRA15-mediated TRAF6 recruitment mediates the recruitment of immunity-related GTPases and destruction of the vacuole. Thus, we have identified how the Toxoplasma effector GRA15 affects cell-autonomous immunity in human and murine cells. 


\section{Introduction}

Toxoplasma is a highly successful obligate intracellular parasite that can establish lifelong chronic infections in a wide range of warm-blooded animals. In humans it causes opportunistic infections in immunosuppressed patients, congenital infections (Hill \& Dubey, 2002), and blindness (Pleyer et al, 2014). Many different Toxoplasma strains exist but in Europe and North-America human infections are dominated by the type I and type II clonal lineages (Howe \& Sibley, 1995; Saeij et al, 2005). Upon host cell invasion, Toxoplasma wraps itself with the host cell plasma membrane, which becomes the nascent parasitophorous vacuole membrane. The vacuole membrane does not fuse with the endo-lysosome system and without immune pressure the vacuole does not acidify thus providing Toxoplasma with a niche for replication (Jones et al, 1972; Mordue \& Sibley, 1997).

Like Toxoplasma, many intracellular pathogens reside within a vacuole (pathogen-containing vacuole or PV) in the host cytoplasm (Liehl et al, 2015). The PV membrane (PVM) protects these pathogens from detection by host cytosolic pathogen recognition receptor (PRRs). However, the host has developed mechanisms to destroy the PV thereby exposing the pathogen (Liehl et al, 2015; Saeij \& Frickel, 2017). For example, in mice interferons upregulate the expression of two families of large dynamin-like GTPases: the immunity-related GTPases (IRGs) and the guanylate binding proteins (GBPs) (Howard et al, 2011), which mediate the destruction of the PV of Toxoplasma and of many gram-negative bacteria (Liehl et al, 2015). Once the pathogen is in the cytoplasm, GBPs and IRGs can also mediate the vesiculation of the pathogen itself thereby exposing pathogen associated molecular patterns (PAMPs) to cytosolic PRRs, which can lead to the activation of the inflammasome (Man et al, 2017) and the induction of a form of cell death called pyroptosis (Broz \& Dixit, 2016). Because host cell death removes the replication niche of intracellular pathogens, this is an efficient way of inhibiting pathogen growth (Krishnamurthy et al, 2017).

The mechanism of IRG and GBP recruitment to the Toxoplasma PVM and the identity of Toxoplasma effectors influencing IRG/GBP recruitment, or their activity, are intense areas of research. These GTPases are normally held inactive on host endomembranes by regulatory 'GMS motif' type IRGs (Haldar et al, 2013). Initially, 'pioneer' effector 'GKS' motif type IRGs are recruited to the Toxoplasma PVM(Hunn et al, 2008), which is largely devoid of regulatory IRGs, where they oligomerize and become activated. What exact signal initiates the recruitment of these pioneer IRGs to the PVM is unclear. In murine cells the initial conjugation of a 
bioRxiv preprint doi: https://doi.org/10.1101/2020.02.24.963496; this version posted February 26, 2020. The copyright holder for this preprint (which was not certified by peer review) is the author/funder, who has granted bioRxiv a license to display the preprint in perpetuity. It is made available under aCC-BY-NC-ND 4.0 International license.

ubiquitin-like protein (e.g. microtubule-associated protein light chain 3 (LC3) or y-aminobutyric acid receptorassociated proteins (GABARAPS)) to the PVM was proposed to be the signal that initiates recruitment of 'pioneer' IRGs (Choi et al, 2014; Sasai et al, 2017). PVM recruitment of pioneer IRGs somehow promotes the ubiquitination of the PVM which subsequently leads to the recruitment of the ubiquitin-binding protein p62 (also called sequestosome or SQSTM1) and E3 ubiquitin ligases (e.g. TNF receptor associated factor 6 or TRAF6 and Tripartite motif containing 21 or TRIM21) in a co-dependent manner (Foltz et al, 2017; Haldar et al, 2015; Lee et al, 2015). PVM ubiquitination by these ubiquitin ligases can lead to further recruitment of p62 and TRAF6 thereby creating an amplification loop ensuring the full ubiquitination of the PV. GBPs also get recruited to the PVM and eventually vesiculation of the PVM by GBPs and IRGs expose Toxoplasma, which can lead to its destruction by GBPs (Degrandi et al, 2013; Kravets et al, 2016) and pyro-necrosis of host cells (Zhao et al, 2009). To counter the host defense mechanisms, Toxoplasma secretes ROP and GRA effector proteins into the host cell from organelles called rhoptries and dense granules, respectively. In both murine and human cells, the type II strain is more susceptible to IFNY-mediated growth inhibition than the type I strain (Clough et al, 2016; Haldar et al, 2015; Qin et al, 2017; Selleck et al, 2015). Resistance of type I strains in murine cells is determined primarily by polymorphic ROP5 and ROP18, which, together with ROP17 and GRA7, cooperatively block IRG and GBP loading on the PVM and subsequent events (Behnke et al, 2015; Etheridge et al, 2014; Fleckenstein et al, 2012; Haldar et al, 2015; Niedelman et al, 2012; Steinfeldt et al, 2010; Khaminets et al, 2010). ROP16 and GRA15 also affect GBP loading on the PVM in murine cells through an unknown mechanism (Virreira Winter et al, 2011).

IFNy-stimulated human cells control Toxoplasma using diverse mechanisms dependent on the cell type (Krishnamurthy et al, 2017). For example, IFNy-mediated induction of Indoleamine 2,3 dioxygenase (IDO) causes breakdown of L-Tryptophan, for which Toxoplasma is auxotrophic, which has been shown to mediate inhibition of parasite growth in HeLa, HAP1, and fibroblast cells (Niedelman et al, 2013; Bando et al, 2018; Qin et al, 2017; Pfefferkorn, 1984; Pfefferkorn et al, 1986). In some human cell lines another mechanism for parasite control is ubiquitination of the vacuole, which leads to lysosomal fusion in HUVEC cells, while in HeLa cells an autophagic double membrane forms around the vacuole and parasite growth is stunted without lysosomal fusion (Clough et al, 2016; Selleck et al, 2015). In certain human cells, GBP1 also seems important for restriction of Toxoplasma growth but the mechanism of growth restriction is unclear. In a lung epithelial cell 
line (A549) GBP1 restricts parasite growth without its recruitment to the PVM (Johnston et al, 2016) while in human mesenchymal stem cells (MSC) growth restriction was associated with GBP1 PVM recruitment (Qin et al, 2017). Much less is known about what initiates targeting of human immune effectors to the PVM and how this leads to parasite elimination. In contrast to mice, humans lack IFNy-inducible IRGs, likely explaining why ROP5, ROP18, and ROP17 do not seem to play an important role in conferring protection against IFNymediated growth inhibition in human cells (Clough et al, 2016; Niedelman et al, 2012; Selleck et al, 2015). Currently, no parasite proteins that determine strain differences in susceptibility to IFNy-mediated cell autonomous immunity in human cells have been identified. A secreted parasite effector, Toxoplasma inhibitor of STAT1-dependent transcription (TgIST), which blocks the STAT1 transcriptional response, was recently described but this effector functions upstream of the upregulation of IFNy-induced toxoplasmacidal mechanisms in both type I and type II strains (Gay et al, 2016; Olias et al, 2016).

Herein, we report that the PVM-localized Toxoplasma GRA15 effector (Rosowski et al, 2011) enhances parasite susceptibility to IFNy in primary human foreskin fibroblast (HFFs) and murine embryonic fibroblasts (MEFs). GRA15 binds several ubiquitin ligases, including TRAF2 and TRAF6, which in HFFs is associated with enhanced recruitment of p62, LC3 and GABARAP to the PVM, enhanced endo-lysosomal fusion, and parasite destruction. Unlike what has been described for murine cells, PVM ubiquitination does not correlate with Toxoplasma elimination in IFNy-stimulated HFFs. The type I RH strain, which does not express a functional GRA15, is much less susceptible to IFNy-mediated elimination. In MEFs, GRA15 also interacts with TRAF2 and TRAF6, and TRAF6 recruitment leads to enhanced PVM loading with IRGs and GBPs and parasite destruction. Thus, we determined that the Toxoplasma effector GRA15 mediates strain differences in susceptibility to cell autonomous immunity in human cells and determined the mechanism by which GRA15 enhances parasite susceptibility to host IFNy in both human and murine fibroblasts.

\section{Results}

\section{The polymorphic effector GRA15 enhances Toxoplasma susceptibility to IFNy-mediated growth}

\section{inhibition in HFFs}

To determine if the type I RH and the type II Pru strain differ in susceptibility to IFNy-mediated growth inhibition in HFFs, we measured the IFNy-mediated reduction in plaque numbers and plaque area. The relative 
reduction in the number of plaques formed in IFNY-stimulated $v s$. unstimulated cells reflects killing of Toxoplasma, while the relative reduction in the area of the plaques is a sensitive assay that reflects overall inhibition of parasite growth over multiple lysis cycles (Niedelman et al, 2012, 2013). Compared to $\mathrm{RH}, \mathrm{Pru}$ had a larger IFNy-induced loss in plaque numbers and plaque area (Fig. 1a). We used parasites expressing luciferase to determine IFNy-mediated growth inhibition $24 \mathrm{~h}$ after infection and observed that Pru growth was more inhibited by IFNy compared to $\mathrm{RH}$ growth (Fig. 1b). IFNy stimulation resulted in a decrease in the relative number of vacuoles (Fig. 1c) as well as a decrease in the number of parasites per vacuole (Fig. 1d), both of which were more pronounced for the Pru strain.

After invasion, Toxoplasma resides within the host cytosol in a PV and starts secreting GRAs into the PV lumen where they stay or get transported to the PVM or beyond the PVM into the host cell (Hakimi et al, 2017). The transport of GRAs beyond the PVM, but not onto the PVM, is mediated by a putative translocon containing the proteins MYR1/2/3 (Franco et al, 2016; Naor et al, 2018). However, Pru and Pru $\Delta m y r 1$ parasites showed similar IFNy-mediated reductions in plaque number and area (Suppl. Fig. 1a) indicating that these phenotypes are not influenced by GRAs secreted beyond the PVM. We therefore hypothesized that maybe a GRA in the PVM facing the host cytosol might be involved. GRA15 is a polymorphic Toxoplasma effector protein present in the PVM that activates the NF-kB transcription factor (Rosowski et al, 2011), a master regulator of cell signaling and cell death (Dutta et al, 2006), independent of MYR1 (Franco et al, 2016). The type I RH strain has an early stop codon in GRA15 leading to a non-functional GRA15 (Rosowski et al, 2011). To determine if GRA15 plays a role in the susceptibility of Pru to IFNy-mediated growth inhibition, we infected IFNy-stimulated or naïve HFFs with Pru, Pru $\Delta g r a 15$, and the Pru $\Delta g r a 15$ strain complemented with an HAtagged copy of GRA15 (Pru $\Delta g r a 15+G R A 15)$ and measured plaque number and area after 6 days. Pru $\Delta$ gra 15 parasites showed significantly less plaque number and area loss compared to wild-type parasites in IFNystimulated HFFs (Fig. 1e). Complementation of $\Delta$ gra15 parasites with GRA15 restored growth inhibition to wild-type levels (Fig. 1e). RH parasites expressing type II GRA15 (RH+GRA15॥) (Rosowski et al, 2011) showed significantly more plaque loss compared to the wild-type $\mathrm{RH}$ strain (Fig. 1f). Furthermore, we observed that the enhanced GRA15-mediated susceptibility of Pru to IFNy was already apparent $24 \mathrm{~h}$ post infection (p.i.) (Fig. 1g) and GRA15 also enhanced the IFNy-mediated elimination of vacuoles (Fig. 1h). 
bioRxiv preprint doi: https://doi.org/10.1101/2020.02.24.963496; this version posted February 26, 2020. The copyright holder for this preprint

(which was not certified by peer review) is the author/funder, who has granted bioRxiv a license to display the preprint in perpetuity. It is made available under aCC-BY-NC-ND 4.0 International license.

It was recently shown that in immortalized HFFs, IFNY-induced IDO1 expression determines the IFNYmediated growth inhibition of Toxoplasma. The MYR1-dependent secreted Toxoplasma effector TgIST was shown to protect Toxoplasma from IDO1-mediated growth inhibition in cells stimulated after infection by inhibiting STAT1-mediated IDO1 expression but not in cells pre-stimulated with IFNy (Bando et al, 2018). In contrast, we previously showed that IDO-mediated L-Trp degradation only plays a minor role in inhibition of parasite growth in IFNy-stimulated primary HFFs (Niedelman et al, 2013). To rule out the role of IDO in the increased susceptibility of Pru parasites to IFNy we show that L-Trp supplementation did not restore the reduction of plaque loss in $\mathrm{RH}$ and Pru strains (Suppl. Fig. 1b). Furthermore, there is no difference in IDO activity in RH vs. Pru-infected IFNy-stimulated HFFs (Suppl. Fig. 1c). Thus, in IFNy-stimulated HFFs parasite expression of GRA15 leads to reduced parasite growth and enhanced disappearance of vacuoles.

\section{GRA15 enhances IFNy-induced endo-Iysosomal fusion with the vacuole}

In HUVEC cells, ubiquitin, ubiquitin-like proteins (LC3/GABARAP), and ubiquitin receptors (p62/NDP52) are recruited to the Toxoplasma PVM, eventually leading to its destruction by fusion with endo-lysosomes (Clough et al, 2016). To determine if this happens in HFFs and the potential role of GRA15 in this process, we pre-stimulated HFFs with IFNy and infected cells with RH, Pru or Pru $\Delta$ gra 15 parasites and measured accumulation of ubiquitin, p62, NDP52, LC3B, GABARAP and LAMP1 around the PVM 3 h p.i. Surprisingly, and unlike what has been observed in HeLa, HUVEC and murine cells (Clough et al, 2016; Haldar et al, 2015; Lee et al, 2015; Selleck et al, 2015), we observed that although PVMs of both RH and Pru strains were coated with ubiquitin in IFNy-stimulated HFFs, a larger fraction of $\mathrm{RH}$ vacuoles was coated (Fig. 2a). We did not observe any difference in the ubiquitin coating intensity among the different parasite strains (Suppl. Fig. 2a). Deletion of GRA15 had no effect on ubiquitination of Pru vacuoles (Fig. 2a). The type of ubiquitin linkage recruited to the PVM can influence the subsequent outcome (Swatek \& Komander, 2016). We observed K63linked, and no K48-linked (not shown), ubiquitin localized to the PVM (Fig. 2b). We observed a significantly larger fraction of Pru PVMs coated with p62 compared to RH (Fig. 2c). Deletion of GRA15 from Pru resulted in significant fewer PVMs coated with p62 while a similar fraction of PVMs of the GRA15 complemented strains as wild-type Pru were coated with p62 (Fig. 2c). Unlike the type I RH strain, the type I GT1 strain contains a functional GRA15, which we previously showed determines $\mathrm{RH}$ vs. GT1 differences in activation of NF-KB 
(Yang et al, 2013). Consistent with a role for GRA15 in mediating p62 PVM-recruitment, a significantly larger fraction of the vacuoles from the $\mathrm{RH}+\mathrm{GRA} 15_{\|}$and the GT1 strain stained positive for p62 compared to $\mathrm{RH}$ vacuoles (Suppl. Fig. 2b). Additionally, we observed that GT1 was significantly more susceptible to IFNYmediated parasite elimination compared to $\mathrm{RH}$ (Suppl. Fig. 2c). A similar fraction of PVMs of $\mathrm{RH}$ and Pru vacuoles contained NDP52 in IFNy-stimulated HFFs (Fig. 2d). However, deletion of GRA15 in Pru resulted in reduction of NDP52 recruitment to the PVM (Fig. 2d). Like p62, both LC3B and GABARAP were recruited to a larger fraction of the PVs of Pru compared to $\mathrm{RH}$ (Fig. 2e and f) in IFNY-stimulated HFFs. The Pru $\Delta$ gra15 strain had 2-fold less vacuoles that were coated with LC3B and GABARAP compared to wild-type Pru (Fig. 2e and f). The GRA15 complemented strain had a larger fraction of PVs coated with LC3B compared to the GRA15 deleted strain (Fig. 2e). To determine if the recruitment of LC3B, GABARAP and p62 is associated with lysosomal destruction of the vacuole we infected IFNY-stimulated HFFs and counted LAMP1-positive vacuoles $3 \mathrm{~h}$ p.i. IFNy enhanced the recruitment of LAMP1 to vacuoles of all strains but significantly more LAMP1-positive vacuoles were seen in Pru-infected, compared to $\mathrm{RH}$-infected, cells. Deletion of GRA15 significantly reduced the number of LAMP1-positive vacuoles (Fig. 2g). In many LAMP1-positive vacuoles the parasites were distorted, and they often did not stain positive for GRA7, used as parasite PV marker. However, by using the DNA-binding dye Hoechst these vacuoles still clearly contained parasite DNA but were in advanced stages of parasite degradation (Suppl. Fig. 3). The lysosomal inhibitor BafA1 significantly inhibited the disappearance of vacuoles in IFNy-stimulated cells (Fig. $\mathbf{2 h}$ ).

Overall these results indicate that GRA15 enhances vacuole destruction via endo-lysosomal fusion in IFNystimulated HFFs.

\section{GRA15-mediated enhancement of destruction of Pru vacuoles through endo-lysosomal fusion in IFNy-} stimulated HFFs correlates with PVM recruitment of p62, LC3B, GABARAP, and LAMP1 but not

\section{ubiquitin}

In IFNy-stimulated HUVEC cells only type II strain PVMs are ubiquitinated and this ubiquitination is indispensable for subsequent endo-lysosomal fusion and parasite elimination (Clough et al, 2016). However, in HFFs, strain differences in ubiquitination of the PVM (Fig. 2a, 2b) did not correlate with recruitment of p62, LC3B, GABARAP and LAMP1 to the PVM (Fig. 2c-g). Furthermore, when we inhibited ubiquitination using a 
specific inhibitor of E1 ubiquitin activating enzymes (PYR 41), we observed a significant reduction in the

fraction of PVMs coated with ubiquitin but no effect on the fraction of PVMs coated with p62 or LC3B (Fig. 3a).

Thus, the fraction of PVMs containing p62 or LC3B does not correlate with the fraction of PVMs containing ubiquitin. Consistent with this, only $25 \%$ of the vacuoles were coated with both ubiquitin and p62, only $40 \%$ of the vacuoles were coated with both ubiquitin and LC3B and only $20 \%$ of the vacuoles were coated with ubiquitin and GABARAP (Fig. 3b-d). In contrast, 90\% of the vacuoles were coated with p62 and LC3B (Fig.

3e) and $78 \%$ with p62 and GABARAP (Fig. 3f). Given that $\sim 20 \%$ of the vacuoles that are coated with only p62 and $\sim 60 \%$ are only Ub positive (Fig. 3b) these data suggest that recruitment of p62 to the PVM is not dependent on PVM ubiquitination.

\section{GRA15 binds TRAFs and recruits TRAF6 and ubiquitin-like molecules and receptors to the} parasitophorous vacuole to mediate parasite elimination

We wanted to determine if GRA15 inhibits parasite growth in IFNy-stimulated HFFs through its ability to activate the NF-KB transcription factor (Rosowski et al, 2011). To test this, we treated HFFs with BAY11-7082, a known inhibitor of NF-KB activation (García et al, 2005), 2 h before infection and 24 h p.i. measured parasite growth. Addition of BAY11-7082 $(1 \mu \mathrm{M})$ did not restore parasite growth in IFNY-stimulated HFFs (Fig. 4a). At this concentration, BAY11-7082 can successfully inhibit the activation of NF-kB triggered by Pru parasites (Fig. 4b). BAY11-7082 was reported to inhibit NF-KB activation via inhibition of E2 ubiquitin conjugating enzymes that mediate K63- and linear polyubiquitin chains (Strickson et al, 2013). We therefore also counted the percentage of PVs coated with ubiquitin or p62 in IFNy-stimulated HFFs and observed that although treatment with BAY11-7082 decreased the fraction of PVs that were coated with ubiquitin, it had no effect on the fraction of PVs coated with p62 (Suppl. Fig. 4a).

To identify the mechanism by which GRA15 enhanced the recruitment of ubiquitin-like molecules and ubiquitin receptors to the PVM, we immunoprecipitated GRA15 from naive and IFNy-stimulated HFFs 8 h p.i. As a control we immunoprecipitated GRA35, which we and others recently showed is another PVM-localized GRA (Nadipuram et al, 2016; Wang et al, 2019b). GRA15 specifically immunoprecipitated multiple ubiquitin ligases, TRAF1, TRAF2, BIRC2, BIRC3, and TNFAIP3 (also named A20), while in IFNy-stimulated cells also polyubiquitin and TRAF6 were immunoprecipitated (Suppl. Table 2). To confirm some of these results, we 
immunoprecipitated GRA15 and blotted for TRAF2 and TRAF6. Indeed GRA15, but not GRA35,

immunoprecipitated TRAF2 in both stimulated and unstimulated HFFs while a small amount of TRAF6 was only immunoprecipitated in IFNy-stimulated HFFs (Fig. 4c). We also observed a significantly larger fraction of Pru PVs coated with TRAF6 compared to RH PVs in IFNY-stimulated HFFs (Fig. 4d). Pru $\Delta$ gra15 PVMs had less recruitment of TRAF6 compared to the wild-type Pru strain (Fig. 4d). However, this antibody seemed to have quite some background staining. TRAF6 is a ring type E3 ubiquitin ligase and catalyzes the formation of K63-linked ubiquitination (Deng et al, 2000) and was reported to be associated with ubiquitination of PVs in murine cells (Haldar et al, 2015). We observed that although inhibition of TRAF6 ubiquitin ligase activity significantly lowered K63-linked ubiquitination on the PVM this had no effect on recruitment of p62 to the PVM (Suppl. Fig. 4b). Because inhibiting ubiquitin activating (E1), ubiquitin conjugating (E2) and ubiquitin ligase (E3) activity did not affect p62 recruitment, if TRAF6 has a role in vacuole destruction it is unlikely to be the ubiquitination of the PVM but more likely the recruitment of p62 via its p62 binding domain. Recently, we have reported that activation of NF-KB by GRA15 depends on in its interaction with TRAF2 as well as TRAF6 in HEK cells (Sangaré et al, 2019). GRA15 has 2 binding motifs for TRAF2 and one for TRAF6 (Suppl. Fig. 5a) and contains at least 4 high confidence ubiquitination sites (Suppl. Fig. 5b). However, none of the ubiquitination sites have the consensus downstream p62-TRAF6 binding sites (Suppl. Fig. 5c). To directly determine the relevance of the GRA15 interaction with TRAF2 and TRAF6 at the PVM, we generated RH parasites that expressed either HA-tagged wild-type GRA15 (RH+GRA15 $\left.{ }_{W T}\right)$ or GRA15 with mutated TRAF2(RH+GRA15 TRAF2mut $)$ or TRAF2- and TRAF6-binding sites ( $\mathrm{RH}+\mathrm{GRA15}$ TRAF2/6mut $)$ Immunofluorescence and Western blot analysis showed that GRA15 had a similar PV/PVM localization in RH+GRA15 ${ }_{W T}$ RH+GRA15 TRAF2mut, and $\mathrm{RH}+\mathrm{GRA15}_{\text {TRAF2/6mut }}$ and had similar expression levels in these parasites (Suppl. Fig. 5e and f). We immunoprecipitated GRA15 from these different parasites from lysates generated from naive or IFNy-stimulated infected HFFs 8 h p. i. Immunoprecipitated GRA15wT pulled down TRAF2 from infected HFF lysates while no TRAF2 was pulled down after immunoprecipitation of GRA15 ${ }_{\text {TRAF2mut }}$ or RH+GRA15 $5_{\text {TRAF2/6mut. }}$ In the lysate from IFNY-stimulated infected HFFs, GRA15 ${ }_{W T}$ pulled down TRAF2 and TRAF6 but no TRAF2 or TRAF6 was pulled down after immunoprecipitation of GRA15 TRAF2/6mut $_{\text {and no TRAF2 and significantly less }}$ TRAF6 was pulled down after immunoprecipitation of GRA15TRAF2mut. These data confirmed that mutation of the respective TRAF2/6-binding sites indeed abrogated the binding to TRAF2 or to TRAF2 and TRAF6 (Fig. 
bioRxiv preprint doi: https://doi.org/10.1101/2020.02.24.963496; this version posted February 26, 2020. The copyright holder for this preprint (which was not certified by peer review) is the author/funder, who has granted bioRxiv a license to display the preprint in perpetuity. It is made available under aCC-BY-NC-ND 4.0 International license.

4e, Suppl. Fig. 5d). The fact that less TRAF6 was immunoprecipitated with the GRA15 TRAF2mut $_{\text {compared to }}$ GRA15 ${ }_{W T}$ could indicate that TRAF6 was coming down via binding to TRAF2(Davies et al, 2005). We observed

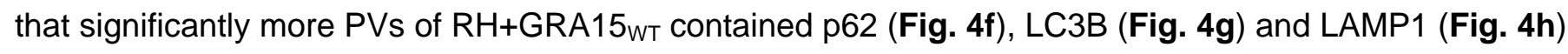
compared to the PVs of $\mathrm{RH}+\mathrm{GRA15}$ TRAF2mut parasites. Recruitment of p62, LC3B and LAMP1 to the PVM of

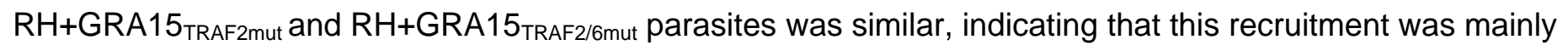
mediated via the GRA15 TRAF2-binding sites (Fig. 4f-h). Furthermore, IFNy-mediated killing of RH+GRA15WT was significantly higher compared to $\mathrm{RH}+\mathrm{GRA} 15_{\text {TRAF2mut }}$ or $\mathrm{RH}+\mathrm{GRA15}$ TRAF2/6mut parasites (Fig. 4i).

Because TRAF2 does not contain a p62-binding domain we hypothesized that its main role was the recruitment of TRAF6. To determine the role of TRAF6, we knocked down TRAF6 in HFFs, using a stable lentivirus mediated siRNA system (Fig. 4j). The growth of Pru parasites in presence of IFNy in these TRAF6 knockdown HFFs was significantly restored compared to scrambled siRNA transfected HFFs (Fig. 4k) whereas both $\mathrm{RH}$ and Pru $\Delta$ gra 15 were resistant in both cell types (Fig. 4k). Furthermore, recruitment of p62 and LC3B in IFNy-stimulated cells was significantly less in TRAF6 knockdown HFFs compared to scrambled siRNA transfected HFFs (Fig. $\mathbf{4 I}$ and $\mathbf{m}$ ). These results indicate that GRA15 by binding with ubiquitin ligases TRAF2 and TRAF6 mediates the susceptibility of type II Pru strains in IFNy-stimulated primary human fibroblasts.

\section{GRA15 mediates susceptibility of type II parasites to IFNy-mediated killing in murine fibroblasts by}

\section{binding to TRAF6}

Our results show that GRA15 mediates Pru susceptibility to IFNy in HFFs by enhancing lysosomal destruction of the vacuole. In IFNY-stimulated MEFs, type II GRA15 plays a role in recruitment of GBPs to the PVM via an unknown mechanism (Fisch et al, 2019; Virreira Winter et al, 2011). In IFNy-stimulated MEFs, GBP recruitment is initiated by PVM recruitment of so-called 'pioneer' IRGs such as IRGB6 (Haldar et al, 2015). In MEFs recruitment of TRAF6 and TRIM21, two ubiquitin ligases, to the PVM enhances subsequent recruitment of IRGs and GBPs that target the vacuole for degradation (Haldar et al, 2015; Foltz et al, 2017). We hypothesized that TRAF6 recruitment by GRA15 might mediate the recruitment of GBPs and IRGs as TRAF6 has a p62 binding motif and p62 has an LC3 interacting region (LIR) motif that can mediate recruitment of LC3B and GABARAP (Lamark et al, 2017). To determine the role of GRA15 in MEFs, we infected IFNy-stimulated MEFs 
bioRxiv preprint doi: https://doi.org/10.1101/2020.02.24.963496; this version posted February 26, 2020. The copyright holder for this preprint (which was not certified by peer review) is the author/funder, who has granted bioRxiv a license to display the preprint in perpetuity. It is made available under aCC-BY-NC-ND 4.0 International license.

and measured PV recruitment of TRAF6, IRGB6, ubiquitin (K63 and K48), GBPs, and LC3B. As demonstrated by others (Foltz et al, 2017; Haldar et al, 2015), Pru parasites had a significantly larger fraction of its vacuoles coated with these markers compared to $\mathrm{RH}$ parasites. This enhanced PVM recruitment was partially mediated by GRA15 as we observed significantly fewer PVs coated with these markers in Pru $\Delta$ gra15 parasites (Fig. 5af). In contrast to what we observed in HFFs, significantly more Pru vacuoles were coated with ubiquitin, predominantly K63-linked, in IFNy-stimulated MEFs compared to $\mathrm{RH}$ vacuoles which is in agreement with previous studies (Foltz et al, 2017; Haldar et al, 2015). We determined parasite growth in IFNy-stimulated MEFs $24 \mathrm{~h}$ p.i. and observed that Pru $\Delta$ gra15 had significantly less IFNy-mediated growth reduction compared to either wild type or GRA15 complemented parasites (Fig. 5g). Pru $\Delta$ gra 15 also showed significantly less plaque loss compared to its parental or complemented strains (Fig. 5h). RH expressing type II GRA15 formed less and smaller plaques compared to wild-type $\mathrm{RH}$ in IFNY-stimulated MEFs (Fig. 5i-j).

To establish that the GRA15-enhanced recruitment of these markers to the vacuole could be mediated by its interaction with TRAF6, we immunoprecipitated GRA15 from naïve and IFNY-stimulated MEFs and performed Western blot for TRAF2 and TRAF6. We observed that akin to HFFs, GRA15 in MEFs also binds TRAF2 and TRAF6 (Fig. 6a). We compared recruitment of TRAF6 to PVs of RH, Pru and Pru $\triangle$ gra15 in MEFs. Akin to HFFs, we observed that in IFNy-stimulated MEFs, significantly more Pru PVs contained TRAF6 (3-fold increase) compared to either RH or Pru $\Delta$ gra15 PVs (Fig. 6b). Significantly fewer Pru PVs were coated (3-fold decrease) with IRGB6 in in Traf6 ${ }^{--}$MEFs compared to wild-type MEFs (Fig. 6c). Furthermore, the difference in IRGB6 coating between Pru and PruAgra15 PVMs in wild-type MEFs disappeared in Traf6/- MEFs (Fig. 6c). We also measured the growth of Pru, Pru $\Delta g r a 15$ and GRA15 complemented parasites in wild type, Traf6 $^{/-}$, and NF-kB $p 65^{/-}$MEFs 24 h p.i. In wild type and NF-kB $p 65^{\prime-}$ MEFs both Pru and GRA15 complemented parasites showed a significant growth reduction upon IFNY-stimulation (Fig. 6d). In contrast, in Traf6 ${ }^{/-}$MEFs, $^{-}$ the action of IFNy was abolished and GRA15-expressing parasites no longer showed reduced growth compared to the Pru $\Delta$ gra15 strain, which was resistant to IFNy in all MEFs (Fig. 6d). To further establish the role of GRA15-mediated TRAF6 binding in parasite growth inhibition in IFNy-stimulated MEFs we infected MEFs with $\mathrm{RH}, \mathrm{RH}+\mathrm{GRA15}{ }_{\mathrm{WT}}, \mathrm{RH}+\mathrm{GRA15}$ TRAF2mut $_{\text {or }} \mathrm{RH}+\mathrm{GRA15} 5_{\text {TRAF2/6mut }}$ and enumerated the vacuoles with recruitment of IRGB6, GBP1-5, p62, TRAF6 and ubiquitin. By immunoprecipitation of GRA15 and Western blotting for TRAF2 and TRAF6, we demonstrated that these mutants are indeed unable to bind TRAF2 or 
TRAF2 and TRAF6 (Fig. 6e). Furthermore, as expected, the $\mathrm{RH}+\mathrm{GRA15}$ TRAF2/6mut strain no longer recruited

TRAF6 to the vacuole while TRAF6 recruitment to the PVM of $\mathrm{RH}+\mathrm{GRA} 15_{\text {TRAF2mut }}$ was similar to $\mathrm{RH}+\mathrm{GRA15}$ WT

(Fig. 6f). The reduced recruitment of TRAF6 to the PVM of the $\mathrm{RH}+\mathrm{GRA} 15_{\mathrm{TRAF} 2 / 6 \text { mut }}$ strain was associated with a reduced recruitment of IRGB6, GBP1-5 and p62 (Figure $6 \mathrm{~g}-\mathbf{i}$ ). In contrast, mutating the TRAF2 or the TRAF2 and TRAF6 binding site on GRA15 did not affect ubiquitin coating of the PVM (Fig. 6j) suggesting that although GRA15 expression is associated with increased ubiquitin coating (Fig. 5c, d) this is not mediated by binding of TRAF6 or TRAF2 to GRA15. The RH+GRA15 TRAF2/6mut strain was also less susceptible to IFNy compared to either the $\mathrm{RH}+\mathrm{GRA} 15_{\mathrm{WT}}, \mathrm{RH}+\mathrm{GRA} 15_{\text {TRAF2mut }}$ strains as indicated by reduced plaque loss upon IFNy-stimulation (Fig. 6k).

Thus, GRA15, by recruiting TRAF6 in IFNY-stimulated cells, and not by activating NF-KB p65, enhanced parasite susceptibility to IRG/GBP-dependent elimination in MEFs.

\section{Discussion}

Toxoplasma strain-dependent susceptibility to IFNy in different human and murine cells in vitro is well established (Bando et al, 2018; Clough et al, 2016; Haldar et al, 2015; Niedelman et al, 2012; Qin et al, 2017; Selleck et al, 2013, 2015; Niedelman et al, 2013). However, Toxoplasma effectors that affect strain differences in susceptibility to IFNy-induced cell autonomous immunity in human cells have not been described. We show that in IFNy-stimulated primary HFFs the Toxoplasma effector GRA15 (Jensen et al, 2011; Rosowski et al, 2011) mediates the recruitment of the E3 ubiquitin ligase TRAF6, its binding partner p62 and LC3B and GABARAPs, eventually leading to endo-lysosomal fusion with the vacuole and parasite elimination.

It was previously shown that in murine cells Toxoplasma GRA15 enhances the recruitment of GBP1-5 to the vacuole (Fisch et al, 2019; Virreira Winter et al, 2011). However, it was a mystery how GRA15 mediated this recruitment of GBP1-5 as its only known function was the activation of the NF-kB transcription factor, which does not take place until four hours after infection, while its effect on GBP recruitment can be seen within 1 hour (Rosowski et al, 2011; Virreira Winter et al, 2011). Here we solve this mystery by showing that in both HFFs and MEFs, GRA15 enhances the recruitment of TRAF6 to the PVM. TRAF6 was previously shown to be required for subsequent recruitment of p62, further ubiquitination of the vacuole and its eventual destruction by IRGs and GBPs (Haldar et al, 2015). We confirmed those data and additionally showed that in 
Traf6 $^{--}$MEFs the difference in susceptibility between Pru and Pru $\Delta g r a 15$ disappears further confirming that the effect of GRA15 is mediated through TRAF6. Thus, GRA15 enhances IFNy-mediated parasite elimination in both HFFs and MEFs although the exact mechanism of vacuole elimination is different (Fig. 7).

Previously it was shown that ubiquitination of the PVM is a strictly strain-dependent phenomenon (Clough et al, 2016; Haldar et al, 2015; Lee et al, 2015; Selleck et al, 2015) where initial ubiquitination recruits p62 which further recruits the E3 ubiquitin ligases TRAF6 and TRIM21 to generate an amplification loop that recruits further p62 and LC3 eventually controlling parasite growth (Foltz et al, 2017; Haldar et al, 2015). However, our data show that in contrast to what has been observed in MEFs, PVM ubiquitination is not strain specific in HFFs, inhibition of PVM ubiquitination has no effect on p62 or LC3B recruitment, and a significant fraction of p62-coated PVMs do not contain ubiquitin. Also, in MEFs infected with $\mathrm{RH}$ or $\mathrm{RH}$ parasites expressing wild-type GRA15 or GRA15 TRAF-binding mutants the recruitment of ubiquitin to the PVM did not correlate with recruitment of TRAF6, P62, IRGB6 or GBPs. Our data suggest that the PVM-localized GRA15 parasite effector recruits TRAF6, which then likely further recruits adaptor proteins that in other cell types appear to be recruited by PVM ubiquitination (Clough et al, 2016; Selleck et al, 2015). In HeLa cells the recruitment of p62 and other markers caused parasite growth stunting but no vacuole destruction (Selleck et al, 2015) whereas in HUVEC cells, vacuole destruction by endo-lysosomal fusion was described to be ubiquitin and p62 dependent (Clough et al, 2016). In HFFs we showed that p62 is recruited to the PVM, possibly via its TRAF6-binding domain, and parasite vacuole destruction occurs through endo-lysosomal fusion. What determines the differences between these different cell types is currently unknown.

Although in MEFs GRA15 significantly increased the percentage of vacuoles targeted by IRGB6, vacuoles of the Pru $\Delta$ gra15 strain were still significantly more targeted compared to the $\mathrm{RH}$ strain. This is likely due to strain differences in ROP5, as we and others previously showed that II strains have ROP5 alleles that are less effective at inhibiting IRG loading onto the PVM and that expression of type I or type III ROP5 alleles significantly reduce coating of type II vacuoles with IRGB6 (Etheridge et al, 2014; Fleckenstein et al, 2012; Niedelman et al, 2012; Virreira Winter et al, 2011). GRA15 also likely explains the difference in IRG coating of vacuoles of different type I strains. For example, the GT1 type I strain has a functional GRA15 while the RH type I strains do not. We previously showed that GT1 GRA15 can activate the NF-kB pathway and that vacuoles of the GT1 strain are significantly more coated with IRGs compared to RH vacuoles (Yang et al, 
2013). Although both $\mathrm{RH}$ and GT1 have a lethal dose of just a single parasite in most laboratory mouse strains, deletion of ROP18 makes GT1 avirulent after low dose infection (Shen et al, 2014) while deletion of $R O P 18$ in $\mathrm{RH}$ only delays death of the mice (Alaganan et al, 2014; Shen et al, 2014). It is likely that this difference is mediated by GRA15 as the presence of GRA15 in GT1 would make it more susceptible to IRGB6 PVM coating and subsequent parasite elimination.

Other studies have shown that IFNy-dependent induction of IDO expression plays a role in inhibiting Toxoplasma growth in human fibroblasts (Bando et al, 2018; Pfefferkorn, 1984; Pfefferkorn et al, 1986). However, we find that inhibition of parasite growth is minimally dependent on IFNy-dependent L-Trp breakdown (this study and Niedelman et al., 2013) (Niedelman et al, 2013). These differences might be due to fibroblasts derived from different tissues and/or the use of primary vs. transformed fibroblast (Bando et al, 2018), as the origin of the fibroblasts in other studies is unclear (Pfefferkorn, 1984; Pfefferkorn et al, 1986).

Thus, although we previously thought that the main role of GRA15 was the activation of the NF-KB pathway, it is possible that its primary role is reducing parasite virulence by enhancing IFNy-mediated vacuole destruction. Furthermore, it was recently shown that GRA15 promotes the type I interferon response in mice by mediating STING polyubiquitination and enhanced cGAS/STING signaling through its ability to bind TRAF molecules (Wang et al, 2019a). Taken together, by observing the detrimental effect of GRA15 on the parasite, it might seem disadvantageous for Toxoplasma to have GRA15. However, the rapidly replicating tachyzoite Toxoplasma stages present during acute infection are not orally infectious, in contrast to the slowly replicating encysted bradyzoite stages. Therefore, to enhance its chances of transmission, Toxoplasma needs to balance immune evasion, to enable replication and dissemination, and immune activation, to prevent killing its host before orally infectious tissue cysts are formed. The contrasting goals of immune evasion and immune activation are reflected in Toxoplasma's arsenal of secreted effectors. GRA15 is clearly an effector that makes the parasite less virulent and helps the host survive. In contrast to GRA15, multiple Toxoplasma effectors mediating resistance against IFNy-mediated toxoplasmacidal mechanisms in murine cells have been identified (Hakimi et al, 2017; Hunter \& Sibley, 2012). For example, ROP5 and ROP18, together with ROP17 and GRA7, cooperatively inactive the IRGs and thereby enhance Toxoplasma virulence in mice (Alaganan et al, 2014; Etheridge et al, 2014). However, type III strains and certain atypical strains (P89 and CASTELLS) do not express ROP18 because of a large insertion in their ROP18 promoter region, while the BOF strain has very 
low expression of ROP5 (Niedelman et al, 2012). Similarly, Toxoplasma secretes the effector IST beyond the vacuole and IST inhibits STAT1 transcriptional activity thereby enhancing Toxoplasma virulence (Gay et al, 2016; Olias et al, 2016). However, it also secretes the GRA24 effector beyond the vacuole which activates P38 MAPK thereby activating host immune responses (Braun et al, 2013). The ultimate outcome of infection is therefore determined by the exact combination of these, often polymorphic, effectors and the species or exact genetic background of the host. A combination of effectors that is optimal in one host might kill another host or lead to complete elimination of parasites in yet another host. It is these evolutionary forces that have likely led to differences in GRA15 expression level and/or sequence in different Toxoplasma strains.

In this manuscript, we show how the polymorphic effector GRA15 determines the differential susceptibility of type I RH and type II Pru strains to IFNy-mediated growth inhibition in human and murine cells. This will help to understand the molecular basis of pathogenicity of different Toxoplasma strains in humans.

\section{Materials and Methods}

\section{Reagents and antibodies}

All the reagents, antibodies, primers, gDNAs and siRNAs used in this study are described in detail in Suppl.

\section{Table 1.}

\section{Culture of cells and parasites}

Human foreskin fibroblasts (HFFs) were routinely maintained in DMEM with high glucose (Gibco, Invitrogen) supplemented with $10 \%$ FBS, L-Glutamine $(2 \mathrm{mM}), 100 \mathrm{U} / \mathrm{mL}$ Penicillin, $100 \mu \mathrm{g} / \mathrm{mL}$ Streptomycin and $20 \mu \mathrm{g} / \mathrm{mL}$ Gentamycin (complete medium). HFFs were passed using $0.25 \%$ trypsin. For all the experiments, HFFs were used at passage 5-10, but for serial passage of the parasites, higher passage number of the HFFs were used. Mouse embryonic fibroblasts (MEFs) were maintained in the complete medium supplemented with $10 \mathrm{mM}$ HEPES, $1 \mathrm{mM}$ sodium pyruvate and 1× MEM nonessential amino acids. MEFs were passed using $0.05 \%$ trypsin-EDTA. NF-kB p65 MEFs were a gift from A. Sinai (University of Kentucky College of Medicine, Lexington, KY), and Traf6 ${ }^{--}$MEFs were provided by K. Fitzgerald (University of Massachusetts Medical School, Worcester, MA). All parasite lines were maintained in vitro by serial passage on monolayers of HFFs and cultured in DMEM with high glucose medium supplemented with $1 \%$ FBS, L-Glutamine (2mM), $100 \mathrm{U} / \mathrm{mL}$ Penicillin, $100 \mu \mathrm{g} / \mathrm{mL}$ Streptomycin and $20 \mu \mathrm{g} / \mathrm{mL}$ Gentamycin. A Toxoplasma gondii RH (RH 1-1) strain 
expressing click beetle luciferase and green fluorescence protein (GFP) and a Pru strain expressing firefly luciferase and GFP (Pru $\Delta h p t$, PruA7) were used as representative of type I and type II, respectively (Boyle et al, 2007). A genetically engineered RH strain expressing type II GRA15, Pru $\Delta$ gra 15 and Pru $\Delta g r a 15+$ GRA15HA (GRA15 complemented strain) were described previously (Rosowski et al, 2011).

\section{Generation of TRAF6 knockdown human primary foreskin fibroblasts}

HFFs were seeded in 12 well plates at a concentration of $2 \times 10^{5}$ cells in $2 \mathrm{ml}$ of complete media mentioned above for culturing HFFs, the night before the transduction. The next day, when cells were at $\sim 70 \%$ confluency media were replaced with fresh complete media supplemented with polybrene ( $8 \mu \mathrm{g} / \mathrm{ml}$, EMD Millipore). Ready to use lentivirus particles containing 4 different siRNAs targeting TRAF6 (Suppl. Table 1) or containing scrambled siRNA (Suppl. Table 1) were then used for transfection using 3 different MOls (2/5/10) in 3 different wells for each virus particles (abm Inc, BC, Canada). Cells were then kept at $37^{\circ} \mathrm{C}, 5 \% \mathrm{CO}_{2}$ inside an incubator overnight. The next day, cells were checked under an inverted fluorescence microscope for expression of GFP as the lentivirus expresses GFP as a fusion protein with the puromycin resistance gene and kept for an additional $24 \mathrm{~h}$ in the incubator. Following this, media was replaced with fresh media with puromycin (1.5 $\mu \mathrm{g} / \mathrm{ml}$ ) for $72 \mathrm{~h}$ for selection of the stably transduced cells, at this time puromycin was able to kill all the cells in untransduced cells, seeded in parallel in the same plate. Repeating the selection one more time for another 48 h cells were divided in 6 well plates to check by immunoblot for TRAF6 expression and knockdown of TRAF6 was confirmed.

\section{Generation of MYR1 ko parasite}

To generate the MYR1 insertional mutant in the Pru $\triangle k u 80$ strain, the parasites were co-transfected with a mixture of the pTOXO_Cas9CRISPR:sgMYR1 vector with purified amplicons containing the DHFR cassette flanked by sequences homologous to the sequence targeted by sgMYR1 (5:1 mass ratio). These amplicons were generated by PCR amplification of the DHFR cassette using the primers MYR1-DHFR - F and MYR1DHFR - R, and a vector carrying the DHFR cassette as template (Donald \& Roos, 1993). Stable recombinants were selected with $1 \mu \mathrm{M}$ pyrimethamine, single cell cloned by limiting dilution and verified by PCR analysis. 


\section{GRA15 TRAF-binding site mutant generation.}

GRA15 TRAF2/6 binding site mutation constructs were amplified from pTKO-att-GRA15 „HA vector (Rosowski et al, 2011) using specific primers (see primers Suppl. Table 1) and confirmed by sequencing. $50 \mu \mathrm{g}$ of circular vectors (pTKO-att-GRA15„HA, pTKO-att-GRA15„„-TRA2Fmut-HA and pTKO-att-GRA15„_-TRAF2/6mut-HA) were transfected into $1 \times 10^{7} \mathrm{RH} \Delta h \times g p r t$ parasites by electroporation. Stable integrants were selected in media with $50 \mu \mathrm{g} / \mathrm{ml}$ mycophenolic acid and $50 \mu \mathrm{g} / \mathrm{ml}$ xanthine and cloned by limiting dilution. Expression and correct localization GRA15 to the PVM were confirmed by IFA for HA and Western blotting.

\section{In vitro Toxoplasma infection}

Parasites for in vitro infection were obtained from sequential syringe lysis using $27 \mathrm{G}$ and $30 \mathrm{G}$ needles of heavily infected HFF monolayers followed with a spin at $570 \times \mathrm{g}$ for 7 minutes. For the infection with $\mathrm{RH}$ strains MOls of 1-3 and for Pru MOls of 3-7 were used. Because $\mathrm{RH}$ and Pru strains often differ in viability and infectivity, equivalent 'real' MOls were matched from plaque assay results performed for each experiment to be able to make strain comparisons. Following infection with Toxoplasma, each time plates were centrifuged at $160 \times \mathrm{g}$ for 3 minutes to synchronize the infection, prior to incubation for the required time.

\section{IFNy stimulation of cells}

In most of the experiments, HFFs were stimulated for $18-24 \mathrm{~h}$ in complete medium at $37{ }^{\circ} \mathrm{C}$ with $10 \mathrm{U} / \mathrm{mL}$ of human IFNy (AbD Serotech, stock concentration is 10,000 U/mL). For some experiments, human IFNy was used at concentration of 5-100 U/mL. MEFs were also stimulated for 18-24 h in complete medium with HEPES at $37{ }^{\circ} \mathrm{C}$ with $100 \mathrm{U} / \mathrm{mL}$ murine IFNy (Peprotech, stock concentration is $100,000 \mathrm{U} / \mathrm{mL}$ ).

\section{Use of inhibitors}

For experiments using BAY11-7082 $(1 \mu \mathrm{M})$ or PYR41 $(1 \mu \mathrm{M})$ or C25-140 $(50 \mu \mathrm{M})$, those compounds were added $24 \mathrm{~h}$ post-stimulation with IFNy but $2 \mathrm{~h}$ prior to infection and were kept throughout the infection for BAY11-7082 and C25-140 but washed away just prior to infection in the case of PYR41, as it is toxic to cells after longer incubation times. Bafilomycin A1 (100 nM) was added $1 \mathrm{~h}$ post infection as it affected the parasite invasion process. 


\section{Luciferase assay for parasite growth}

Luciferase assays were performed from the 96 well plates and for each strain and condition, triplicate wells were used. To the confluent monolayers of HFFs/MEFs $\left(2 \times 10^{5}\right.$ cells/well), IFNy was added (10 U/mL of human IFNy and $100 \mathrm{U} / \mathrm{mL}$ of murine IFNy) for $24 \mathrm{~h}$ prior to infection. Next day, infection with indicated parasites strains was done. For each strain 3 different MOls were used for matching of results from similar parasite infectivity between the strains later with the plaque assay. For some experiment's inhibitors were added either $2 \mathrm{~h}$ prior to infection or $1 \mathrm{~h}$ post infection as indicated. Following another $24 \mathrm{~h}$ incubation, culture supernatants were removed and $1 \times$ lysis buffer was added (Luciferase assay system, Promega) to the cells in the wells, followed by three freeze-thaw cycles. After that, $1 \times$ assay buffer containing luciferin was added to each well and transferred to clear centrifuge tubes for measurement of luciferase activity from the lysate using a single channel luminometer (Turner Biosystems). Luciferase reading of wells not treated with IFNy was considered as $100 \%$ and relative growth was calculated for IFNy- and inhibitor-treated wells.

\section{Immunofluorescence assays for recruitment of host markers to the PVM and percentage of infection}

HFFs or MEFs were plated on coverslips in 24 well plates $\left(1 \times 10^{5}\right.$ cells/well) and cultured, stimulated with IFNy for 18-24 h, and subsequently infected with Toxoplasma for $24 \mathrm{~h}$ (to determine the percentage of infected cells and nuclear translocation of NF-kB), $3 \mathrm{~h}$ (to assess the recruitment of ubiquitin, p62, LC3B, GABARAP, GBP2, LAMP1 and TRAF6 in HFFs and MEFs) or $1 \mathrm{~h}$ (to determine IRGB6 coating in MEFs). Following incubation, cells were fixed with either $3 \%$ formaldehyde or $100 \%$ methanol depending on host marker (See Suppl. Table 1) and then permeabilized and blocked with either buffer containing $0.2 \%$ Triton $X-100$ along with $3 \%$ BSA and $5 \%$ goat serum (See Suppl. Table 1) or with buffer containing $0.2 \%$ freshly prepared saponin instead of Triton X-100 (See Suppl. Table 1). Cells were then treated with primary antibodies (Suppl. Table 1) for overnight incubation at $4{ }^{\circ} \mathrm{C}$. Following that, each well was washed 3 times with $1 \times$ PBS and then secondary antibodies were added with Hoechst 33258 for 1 hr. Finally, coverslips were washed 5 times with $1 \times$ PBS and were mounted with VECTASHIELD antifade mounting medium. Imaging was done as described previously (Niedelman et al, 2013). For determination of nuclear translocation of NF-kB, nuclear intensity of at least 15 infected cells were taken into consideration, whereas to assess percentage of infection after $24 \mathrm{~h}$, cells were 
counted in at least 6 independent fields and the values observed in untreated infected cells were taken as $100 \%$ and calculation for the rest was done relative to untreated infected cells. To measure the recruitment of host markers on the PVM, at least 100 infected cells were counted.

\section{Plaque assay}

For the plaque assay, freshly confluent 24 well plates of HFFs or MEFs were used. The day before infection fresh media was added replacing the media from the plates and stimulated with $10 \mathrm{U} / \mathrm{mL}$ human IFNy or 100 $\mathrm{U} / \mathrm{mL}$ mouse IFNy or left unstimulated for $24 \mathrm{~h}$. For infection, freshly harvested parasites, 100 parasites of $\mathrm{RH}$ and 250 parasites of Pru strains were added to the 24 well plates. Infected plates were incubated for 4 days at $37^{\circ} \mathrm{C}$ for $\mathrm{RH}$ strains and for 6 days in the case of Pru strains. For calculating the percentage of plaque loss, the following formula was used as described previously (Niedelman et al, 2012, 2013): [(Number of plaques in unstimulated condition - Number of plaques in stimulated condition)/Number of plaques in unstimulated condition] $\times 100$. Plaque areas were captured and analyzed using a Nikon TE2000 inverted microscope equipped with Hamamatsu ORCA-ER digital camera, and NIS Elements Imaging Software, respectively. Plaque area loss was calculated using the same formula for plaque loss except using the plaque areas in place of plaque numbers. For all experiments, at least 20-25 plaques from technical duplicate wells were imaged.

\section{IDO activity assay}

The IDO activity upon IFNy stimulation and Toxoplasma infection was evaluated by measuring L-Kynurenine from the culture supernatant of HFFs. Cells were cultured in 96-well plates as mentioned in earlier assays with the complete DMEM medium containing total $0.6 \mathrm{mM} \mathrm{L-Tryptophan} \mathrm{(0.52} \mathrm{mM} \mathrm{L-Tryptophan} \mathrm{was} \mathrm{added} \mathrm{to}$ existing $0.08 \mathrm{mM} \mathrm{L-Tryptophan} \mathrm{in} \mathrm{the} \mathrm{media).} \mathrm{HFFs} \mathrm{were} \mathrm{either} \mathrm{stimulated} \mathrm{with} 10 \mathrm{U} / \mathrm{mL}$ IFNy for $18-24 \mathrm{~h}$ or left untreated and subsequently infected with parasite strains at different MOls for $24 \mathrm{~h}$ before harvesting the culture supernatant. The concentration of L-Kynurenine was measured using $1.2 \% \mathrm{p}$ dimethylaminobenzaldehyde in glacial acetic acid solution (Ehrlich reagent). Briefly, $150 \mu \mathrm{L}$ of culture supernatant was mixed with $20 \mu \mathrm{L}$ of $30 \%$ trichloro acetic acid in a V-bottom 96 well plate followed with incubation at $50{ }^{\circ} \mathrm{C}$ for 30 minutes. Subsequently, the plate was centrifuged for 10 minutes at $600 \times \mathrm{g}, 100 \mu \mathrm{L}$ of culture supernatant was mixed with Ehrlich reagent and incubated for 10 minutes and absorbance was 
recorded at $490 \mathrm{~nm}$ using a plate reader (Molecular Device Spctramax M2e). A standard curve of L-kynurenine (0-1500 $\mu \mathrm{M})$ was used to calculate the concentrations in the samples.

\section{Co-Immunoprecipitation:}

40x T175 of human foreskin fibroblast (HFF) were stimulated or not with $10 \mathrm{U} / \mathrm{ml}$ of human IFN-y (AbD

Serotech) for $24 \mathrm{~h}$. Then, $2 \mathrm{~h}$ before infection (MOI:5 to 10) with $\mathrm{RH}+\mathrm{GRA} 15_{\Perp}$-HA or $\mathrm{RH}+\mathrm{GRA35}-\mathrm{HA}$ (for each parasites 10x T175 containing IFN-y stimulated HFFs and 10 unstimulated) the cells were incubated with 50 $\mu \mathrm{M}$ of VX7655 (Selleck chem). $16 \mathrm{~h}$ after infection, the cells were washed once and scraped with cold PBS. The cells were centrifuged and resuspended in $6 \mathrm{ml}$ of lysis buffer (HEPES 10mM pH7.9, $\mathrm{MgCl} 21.5 \mathrm{mM}, \mathrm{KCl}$ 10mM, EDTA $0.1 \mathrm{mM}, 0.5 \mathrm{mM}, \mathrm{NP} 40$ 0.65\%, cocktail of protease inhibitor (Roche), phenylmethylsulfonylfluoride (PMSF) $0.5 \mathrm{mM}$.) for 45 minutes at $4^{\circ} \mathrm{C} .1 \%$ of these lysates were kept as input for the immunoblot. The lysate was centrifuged 30 minutes at $18,000 \times \mathrm{g}, 4^{\circ} \mathrm{C}$. Each sample was incubated with $100 \mu \mathrm{l}$ of $\mathrm{HA}$ magnetic beads (Thermo scientific) rotating overnight at $4^{\circ} \mathrm{C}$. The beads were washed 3 times with Tris- $\mathrm{HCl} 10 \mathrm{mM} \mathrm{pH} 7.5, \mathrm{NaCl}$ 150mM, Triton-100X 0.2\%, PMSF 0.5mM, cocktail of protease inhibitor (Roche), once with Tris- $\mathrm{HCl} 62.5 \mathrm{mM}$ pH 6.8 and subsequently resuspended in $100 \mu \mathrm{l}$ of this buffer performed immunoblotting (30 $\mu \mathrm{l})$ and did the mass spec analysis from the rest of the samples.

To perform immunoprecipitation with GRA15 mutants in HFFs or in MEFs one T175 for each condition (untreated or IFNy-treated) was infected with $\mathrm{RH}+\mathrm{GRA15} \mathrm{WT}_{-}-\mathrm{HA}, \mathrm{RH}+\mathrm{GRA}$ 15 $_{\text {TRAF2mut }}{ }^{-H A}, \mathrm{RH}_{+} \mathrm{GRA}$ 15 $_{\text {TRAF2/6mut }^{-}}$ $\mathrm{HA}$, or $\mathrm{RH}+\mathrm{GRA} 45-\mathrm{HA}$ expressing parasites for $24 \mathrm{~h}$ in MEF and $8 \mathrm{~h}$ in HFFs. Following infection, cells were washed once with ice cold 1x PBS, and then scraped with ice cold PBS, centrifuged and resuspend in $1 \mathrm{ml}$ of lysis buffer mentioned above for 30 minutes at $4^{\circ} \mathrm{C}$. After that $10 \%$ of lysate was put aside for use as input during immunoblotting. The remaining lysates were then processed as mentioned above and incubated with 20 $\mu \mathrm{l}$ of HA magnetic beads overnight, in rotating condition at $4^{\circ} \mathrm{C}$. Next day, beads were washed with the lysis buffer 3 times and subsequently resuspended in $60 \mu$ of $1 x$ loading dye to perform immunoblotting.

\section{Immunoblotting:}

$20 \mu \mathrm{l}$ of the HA magnetic beads of each sample was used to run on a $12 \%$ SDS-PAGE. The proteins were transferred to a PVDF membrane, blocked 30 minutes with TBST, 5\% nonfat dry milk. The membrane was 
blotted overnight at $4^{\circ} \mathrm{C}$ with rat antibody against the HA tag (Roche, 1/500 dilution), TRAF2 and TRAF6 rabbit antibodies (Suppl. Table 1) followed by respective secondary HRP antibodies (Suppl. Table 1).

\section{Mass spectrometry-based proteomics:}

The HA magnetic beads were sent to the Proteomic Core Facility of University of California, Davis for mass spectrometry analysis. Briefly, the proteins were digested using Promega modified trypsin overnight at room temperature on a gently shaking device. Resulting peptides were analyzed by online LC-MS/MS Q-Exactive. All MS/MS samples were analyzed using X! Tandem (The GPM, thegpm.org; version X! Tandem Alanine (2017.2.1.4)). X! Tandem was set up to search the uniprotHSTG_crap database assuming the digestion enzyme trypsin. X! Tandem was searched with a fragment ion mass tolerance of 20 PPM and a parent ion tolerance of 20 PPM. Glu->pyro-Glu of the n-terminus, ammonia-loss of the n-terminus, gln->pyro-Glu of the nterminus, deamidated of asparagine and glutamine, oxidation of methionine and tryptophan, dioxidation of methionine and tryptophan and dicarbamidomethyl of lysine were specified in X! Tandem as variable modifications. Scaffold (version Scaffold_4.8.6, Proteome Software Inc., Portland, OR) was used to validate MS/MS based peptide and protein identifications. Peptide identifications were accepted if they could be established at greater than $50.0 \%$ probability by the Scaffold Local FDR algorithm. Peptide identifications were also required to exceed specific database search engine thresholds and $\mathrm{X}$ ! Tandem identifications were also required. Protein identifications were accepted if they could be established at greater than $9.0 \%$ probability to achieve an FDR less than $5.0 \%$ and contained at least 1 identified peptide. Protein probabilities were assigned by the Protein Prophet algorithm (Nesvizhskii et al, 2003). Proteins that contained similar peptides and could not be differentiated based on MS/MS analysis alone were grouped to satisfy the principles of parsimony. Proteins sharing significant peptide evidence were grouped into clusters.

\section{Statistical analysis}

All statistical analyses were performed using Graph Pad prism version 7.0. All the data presented are mean \pm standard error of mean (SEM) and the exact $n$ values are mentioned in each of the figure legends. For all the calculations, p-values of $<0.05$ are considered as significant. Parameters with 2 different variables and groups were compared by Two-way ANOVA followed with either Bonferroni or Tukey's multiple comparison test. 
Parameters with one variable but three or more groups were compared by One-way ANOVA followed with

Tukey's multiple comparison test. For one variable test with two groups, two-tailed unpaired t-test was used.

Specific statistical test performed for each figure was stated in each of the figure legend.

\section{Data availability}

The authors declare that all data supporting the findings of this study are available within the article and its Supplementary Information files or are available from the authors upon request. All unique materials (e.g. the diversity of parasite lines described in this manuscript) are available upon request (contact: jsaeij@ucdavis.edu).

\section{Acknowledgment}

We thank all members of the Saeij laboratory for productive discussions and Dr. Kevin Woolard for providing the instrumental facilities for assistance in the project. JPJS was supported by the National Institutes of HealthNIH-2R01Al080621-06A1. DM was supported by American Heart Association Post-doctoral fellowship (18POST34030036)

\section{Author contribution}

DM and JPJS designed the study. DM and LOS performed and interpreted the experimental work. LB generated MYR1 knockout parasite strains. MAH provided insightful discussions and constructive suggestions and supervised the generation of knockout parasite strains. JPJS supervised the research. DM and JPJS wrote the paper.

\section{Conflict of interest}

The authors declare that they have no conflict of interest 


\section{Figure legends:}

\section{Figure 1:}

The type II (Pru) Toxoplasma strain is more susceptible to IFNy-mediated growth inhibition in primary human foreskin fibroblasts (HFFs) than the type I RH strain due to presence of the polymorphic effector GRA15.

a) HFFs were pre-stimulated with IFNy $(10 \mathrm{U} / \mathrm{mL})$ for $24 \mathrm{~h}$. Plaque assays were performed for each strain and each condition. Plaque number and area loss were calculated 4 days p.i. for $\mathrm{RH}$ and 6 days p.i. for Pru. Assays were performed with $\mathrm{RH}(n=4)$, Pru $(n=4)$. b) Relative parasite growth was measured 24 h p.i. in IFNystimulated and unstimulated HFFs by luciferase assay. Growth of each strain in IFNy-stimulated HFFs is expressed relative to growth in unstimulated HFFs. Experiments were performed with $\mathrm{RH}(n=4)$, Pru $(n=4)$. c) Percentage of infection was calculated using HFFs grown on coverslips and stimulated with $10 \mathrm{U} / \mathrm{mL}$ IFNy for 24 h. Following stimulation, HFFs were infected with either $\mathrm{RH}(\mathrm{MOI}=1)$ or Pru $(\mathrm{MOI}=3)$ for another $24 \mathrm{~h}$. Coverslips were fixed and stained with GRA7 for parasite PVM and Hoechst 33258 for nuclei. The number of PVs in 5-6 fields of the coverslips were counted for each condition. Experiments were performed with $\mathrm{RH}$ $(n=3)$, Pru ( $n=3)$. d) Parasites per vacuole were determined 24 h p.i. with similar conditions and staining as described in (c) except MOls used were 0.5 and 1 for $\mathrm{RH}(n=3)$ and Pru $(n=3)$, respectively. e) Plaque assays were performed similarly as described for (a) with Pru WT ( $n=20$ for plaque number and $n=7$ for plaque area), Pru $\Delta$ gra15 ( $n=20$ for plaque number and $n=7$ for plaque area), Pru $\Delta g r a 15+G R A 15$ complemented $(n=12$ for plaque number and $n=7$ for plaque area). f) Plaque assays were performed similarly as described for (a) with $\mathrm{RH}$ ( $n=5$ for plaque number and $n=3$ for plaque area) and $\mathrm{RH}+\mathrm{GRA} 15_{\|}$( $n=5$ for plaque number and $n=3$ for plaque area). g) Relative parasite growth was measured as described in (b) with Pru WT ( $n=12)$, Pru $\Delta$ gra15 (n=12), Pru $\Delta$ gra15+GRA15 complemented $(n=8)$. h) Percentage of infection was calculated as described in (c) with Pru WT (n=3), Pru $\Delta g r a 15(n=3), \mathrm{RH}(n=3)$ and $\mathrm{RH}+\mathrm{GRA} 15_{\|}(n=3)$.

Statistical analysis was done by Two-way ANOVA followed by Tukey's multiple comparison test. Data are represented as mean \pm standard error of mean (SEM).

\section{Figure 2:}




\section{GRA15 enhances IFNy-induced PVM decoration with autophagy-related proteins and endo-lysosomal-}

\section{mediated vacuole destruction in HFFs}

a-g) HFFs were stimulated with for $24 \mathrm{~h}$ with $10 \mathrm{U} / \mathrm{mL}$ IFNy or left unstimulated and subsequently infected with

$\mathrm{RH}$, Pru or Pru $\Delta$ gra15 parasites for $3 \mathrm{~h}$. The percentage of vacuoles that stained positive for a) Total ubiquitin ( $n=3$ for $\mathrm{RH}, n=5$ for Pru and $n=3$ for Pru $\Delta$ gra 15), b) K63-linked ubiquitin ( $n=3$ for $\mathrm{RH}, n=3$ for Pru and $n=3$ for Pru $\Delta$ gra15), c) p62 ( $n=3$ for $\mathrm{RH}, n=10$ for Pru and $n=7$ for Pru $\Delta$ gra 15, $n=4$ for Pru $\Delta$ gra 15+GRA15), d) NDP52 ( $n=3$ for $\mathrm{RH}, n=3$ for Pru and $n=3$ for Pru $\Delta$ gra15), e) LC3B ( $n=3$ for $\mathrm{RH}, n=3$ for Pru and $n=3$ for Pru $\Delta$ gra 15 , $n=3$ for Pru $\triangle$ gra 15+GRA15), f) GABARAP ( $n=3$ for $\mathrm{RH}, n=3$ for Pru and $n=3$ for Pru $\Delta g r a 15$ ) and g) LAMP1 ( $n=3$ for $\mathrm{RH}, n=3$ for Pru and $n=3$ for Pru $\Delta$ gra 15) is shown in the left bar diagram. On the right-hand side, a representative fluorescent image is shown for the Toxoplasma Pru strain, which expresses GFP. DNA was stained with Hoechst 33258. Scale bar is $10 \mu \mathrm{m}$. The yellow box inside each representative image is shown as inset pictures with magnification. h) The number of parasite-infected HFFs per 20X objective field was counted and compared between IFNy-stimulated and IFNy + bafilomycin A1 (100 nM) treated HFFs 24 h p.i. with Pru strain. Images from at least 6 fields were taken for each condition $(n=3)$. Each dot represents one experiment. Each time at least 100 different vacuoles were scored and analyzed. Statistical analysis was done by Two-Way ANOVA followed with Tukey's multiple comparison test $(\mathbf{a}-\mathbf{g})$ and One-way ANOVA for $\mathrm{h}$. Data are represented as mean \pm SEM.

\section{Figure 3:}

\section{PVM decoration with autophagy adaptors correlates with p62 but not ubiquitination}

a) HFFs were stimulated with IFNy $(10 \mathrm{U} / \mathrm{mL}$ for $24 \mathrm{~h})$ or left unstimulated and subsequently treated with PYR41 $(1 \mu \mathrm{M})$ for $2 \mathrm{~h}$ prior to infection. Cells were washed and subsequently infected with Pru parasites $(\mathrm{MOI}=3)$ for $3 \mathrm{~h}$. The percentage of vacuoles that stained positive for total ubiquitin, p62 or LC3B was determined $(n=3)$. b-f) For all the co-staining experiments IFNy-stimulated HFFs infected for $3 \mathrm{~h}$ with the Pru strain were used. For each staining at least 50 vacuoles were scored $(n=3)$. On the right-hand side, a representative fluorescent image is shown for the Toxoplasma Pru strain. Scale bar is $10 \mu \mathrm{m}$. The yellow box inside each representative image is shown as inset pictures with higher magnification. The total number of coated vacuoles was set at $100 \%$ and the percentage of vacuoles positive for Ub, p62 and/or LC3B/GABARAP 
was calculated. Statistical analysis was done by One-way ANOVA followed with Tukey's multiple comparison test. Data are represented as mean \pm SEM.

\section{Figure 4:}

\section{GRA15-mediated parasite growth reduction in IFNy-stimulated HFFs is independent of its ability to} activate NF-KB but dependent on GRA15's interaction with the E3 ubiquitin ligases TRAF2 and TRAF6

a) HFFs were stimulated with IFNy for $24 \mathrm{~h}(10 \mathrm{U} / \mathrm{ml})$ or left unstimulated. The NF-KB inhibitor BAY11-7082

$(1 \mu \mathrm{M})$ was added $2 \mathrm{~h}$ pre-infection and HFFs were subsequently infected with Pru parasites. Parasite growth

(using luciferase assay) was measured $24 \mathrm{~h}$ p.i. Means from unstimulated cells were set at $100 \%$. Experiments were performed three times. b) Nuclear translocation of the NF-kB p65 subunit was quantified in HFFs or HFFs treated with BAY11-7082 (1 $\mu \mathrm{M}$, added $2 \mathrm{~h}$ pre-infection) $24 \mathrm{~h}$ p.i. with Pru parasites. Experiments were done 4 times where each dot represents one experimental mean of at least 15 nuclei. In the right panel, representative images are shown. Parasites were expressing GFP, nuclei are stained with Hoechst 33258. Scale bar is 20 $\mu \mathrm{m}$. c) Immunoprecipitation and Western blot were performed in HFFs with and without IFNy (10 U/ml) using an $\mathrm{RH}$ strain expressing type II GRA15-HA and as a control RH expressing GRA35-HA. The blots using antibodies against TRAF2 and TRAF6 were made after stripping the first blot. The inputs loaded represent $1 \%$ of total lysate prepared for immunoblotting and mass spec (Suppl. Table 2). The antibodies against TRAF2 and TRAF6 were obtained from Santa Cruz Biotechnology (Suppl. Table 1). Full length blots for this figure can be observed in supplementary figures. 4c-e. d) Immunofluorescence analysis of TRAF6 recruitment to the PVM of HFFs infected for $3 \mathrm{~h}$ with $\mathrm{RH}$, Pru and Pru $\Delta$ gra15 strains. On the right-hand side, a representative fluorescent image is shown of TRAF6 recruitment to the PVM where Toxoplasma Pru strain expresses GFP, DNA was stained with Hoechst 33258 . Scale bar is $10 \mu \mathrm{m} . n=3$ for all the strains. The antibody against TRAF6 was purchased from Abnova (Suppl. Table 1). e) Immunoprecipitation and Western blot were performed in HFFs

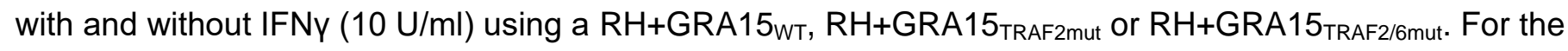
unstimulated cells, uninfected HFFs were used an additional negative control (left panel). Left panel and right panel were run on a single gel; vertical white lines indicate excision of irrelevant lanes. Full length blots are in supplementary figures 4f-k. The antibodies used against TRAF2 and TRAF6 were purchased from Cell Signaling Technology and Abnova, respectively. The asterisks $\left({ }^{*}\right)$ in the lower right panel indicates the faint 


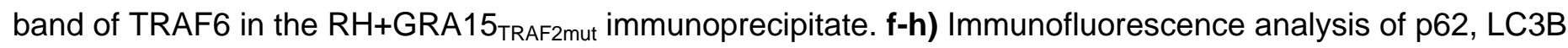
and LAMP1 with and without IFNy in $\mathrm{RH}, \mathrm{RH}+\mathrm{GRA} 15_{\mathrm{WT}}$. All the experiments were done 3 times with each of the strains. i) Plaque assays were performed with $\mathrm{RH}+\mathrm{GRA15}{ }_{\mathrm{WT}}, \mathrm{RH}+\mathrm{GRA} 15_{\text {TRAF2mut }}$ or $\mathrm{RH}+\mathrm{GRA} 5_{\text {TRAF2/6mut }}$ $(n=3)$. j) Expression of TRAF6 was detected by Western blotting of lysates from scrambled siRNA transfected and TRAF6-specific siRNA transfected HFFs with and without IFNy. k) Relative parasite growth was measured in scrambled siRNA transfected HFFs and TRAF6 knockdown HFFs using luciferase based assay with indicated strains with and without IFNy $(n=3)$. The antibody against TRAF6 used here was from Abcam (Suppl. Table 1). Full length blots are in supplementary figures 4I-m. I-m) Immunofluorescence analysis of p62 and LC3B was done in scrambled siRNA transfected HFFs and TRAF6 knockdown HFFs with and without IFNy using indicated strains $(n=3)$. Statistical analysis was done by One-way ANOVA followed with Tukey's multiple comparison test (a, b and i) and two-Way ANOVA followed with Tukey's multiple comparison test (d, f-h and km). Data are represented as mean \pm SEM.

\section{Figure 5:}

\section{GRA15 enhanced susceptibility of type II strains to IFNy-mediated killing in MEFs correlates with} enhanced recruitment of IRGB6, GBPs, Ubiquitin, and LC3B.

MEFs were stimulated with IFNy for 24 h (100 U/ml) a-f) IFNy-stimulated MEFs were infected with Pru, Pru $\Delta$ gra 15 or $\mathrm{RH}$ for $3 \mathrm{~h}$ and subsequently fixed, permeabilized and stained for a) IRGB6, b) GBPs, c) total ubiquitin, d) K63-linked ubiquitin, e) K48-linked ubiquitin and f) LC3B. For analysis, at least 100 vacuoles were scored. All experiments were performed 3 times. On the right-hand side, a representative fluorescent image is shown for the Toxoplasma Pru strain, which expresses GFP. DNA was stained with Hoechst 33258. Scale bar is $10 \mu \mathrm{m}$. The yellow box inside each representative image is shown as an inset picture with magnification. $\mathbf{g}$ ) MEFs were stimulated with IFNy for $24 \mathrm{~h}(100 \mathrm{U} / \mathrm{ml})$ or left unstimulated and subsequently infected with Pru, Pru $\triangle$ gra15 and GRA15 complemented parasites for $24 \mathrm{~h}$ for measuring parasite growth by luciferase assay. All the experiments from a-g were performed 3 independent times. h) Plaque numbers were measured for Pru ( $n=12)$, Pru $\Delta g r a 15$ ( $n=12)$ and GRA15 complemented parasites $(n=6)$. I-j) MEFs were stimulated with IFNy for $24 \mathrm{~h}(100 \mathrm{U} / \mathrm{ml})$ or left unstimulated and subsequently infected with $\mathrm{RH}$ or RH expressing type II GRA15. Plaque numbers were counted $(n=3)$, and areas were measured 4 days p.i. $(n=3)$. Statistical analysis was 
done by One-way ANOVA followed with Tukey's multiple comparison test (a-h), except for (g, j) for which Twoway ANOVA followed with Tukey's multiple comparison test was performed whereas for (i) two sample student's $t$ test was performed. Data are represented as mean \pm SEM.

\section{Figure 6:}

\section{GRA15 enhanced susceptibility of the type II Pru strain in IFNy-stimulated MEFs is dependent on}

\section{TRAF6}

a) Immunoprecipitations and Western blots were performed on MEFs stimulated or not with IFNy (100 U/ml) and infected with an $\mathrm{RH}$ strain expressing type II GRA15-HA and as a control RH expressing GRA45-HA. The blots using antibodies against TRAF6 and TRAF2 were made after stripping the first blot. Left panel and right panel were run on a single gel; vertical white lines indicate excision of irrelevant lanes. Full length blots are in supplementary figures $6 a-c$. The antibodies used against TRAF2 and TRAF6 were purchased from Cell Signaling Technology and Abnova, respectively. b) IFNy-stimulated MEFs were infected with Pru, Pru $\triangle$ gra 15 and $\mathrm{RH}$ for $3 \mathrm{~h}$ and subsequently stained for TRAF6. On the right-hand side, a representative fluorescent image is shown for the Toxoplasma Pru strain, which expresses GFP. DNA was stained with Hoechst 33258. Scale bar is $10 \mu \mathrm{m}$. The yellow box inside each representative image is shown as an inset picture with magnification. Experiments were performed 3 times. c) IRGB6 staining on PVM after infection of wild-type or Traf6 $^{-/-}$MEFs for $3 \mathrm{~h}$ with Pru, Pru $\triangle$ gra15 and $\mathrm{RH}$. On the right-hand side, a representative fluorescent image is shown of IRGB6 coating on the PVM of both wild-type and Traf6 ${ }^{-/-}$MEF. At least 100 different vacuoles were observed and analyzed for each experiment $(n=3)$. d) Parasite growth was measured $24 \mathrm{~h}$ p.i. using luciferase readout from unstimulated and IFNy-stimulated wild-type, NFKB $p 65^{/-}$and Traf6 ${ }^{-1-} \mathrm{MEFs}$. Growth was compared between Pru, Pru $\Delta g r a 15$ and Pru $\Delta g r a 15+G R A 15$ (complemented) strains. Reading from unstimulated cells was considered as $100 \%$ and percentage growth in IFNy-stimulated cells was expressed relative to unstimulated cells. Experiments were performed three times with each MEF type. e) Immunoprecipitation and Western blot were performed in MEFs infected with $\mathrm{RH}+\mathrm{GRA} 15_{\mathrm{WT}}$, $\mathrm{RH}+\mathrm{GRA15}$ TRAF2mut or $\mathrm{RH}+\mathrm{GRA15}$ TRAF2/6mut $_{\text {and }}$ as a control RH expressing GRA45-HA. Left panel and right panel were run on a single gel; vertical white lines indicate excision of irrelevant lanes. Full length blots are in supplementary figures $6 \mathrm{~d}-\mathrm{f}$. The antibodies used against TRAF2 and TRAF6 were purchased from Cell 
Signaling Technology and Abnova, respectively. f-j) Immunofluorescence analysis of TRAF6, IRGB6, GBP1-5, p62 and ubiquitin was done in IFNy-stimulated MEFs infected with $\mathrm{RH}+\mathrm{GRA} 15_{\mathrm{WT}}, \mathrm{RH}+\mathrm{GRA} 5_{\mathrm{TRAF} 2 \mathrm{mut}}$ or $\mathrm{RH}+\mathrm{GRA15}$ TRAF2/6mut $(n=3)$. k) Plaque assays were performed with $\mathrm{RH}+\mathrm{GRA15}{ }_{\mathrm{WT}}, \mathrm{RH}+\mathrm{GRA15}$ TRAF2mut or $\mathrm{RH}+\mathrm{GRA} 15_{\text {TRAF2/6mut }}(n=4)$. Statistical analysis was done by two samples student's $\mathrm{t}$ test (f-j) and One-way ANOVA followed with Tukey's multiple comparison test for $b$ and $k$ and Two-way ANOVA for $c$ and d. Data are represented as mean \pm SEM.

\section{Figure 7:}

\section{Proposed model of GRA15-mediated susceptibility to IFNy-induced growth inhibition in human and} murine fibroblasts.

a) In HFFs, IFNY-stimulation causes recruitment of TRAF2/6 to GRA15 on the PVM of Toxoplasma. What determines the IFNy-induced binding of TRAF6 to GRA15 is unknown, although our results indicate TRAF2 plays a role in this. (i) Recruitment of TRAF6 mediates further recruitment of p62, LC3B and GABARAP. This subsequently leads to endo-lysosomal fusion with the PVM and elimination of the parasites. (ii) On the other hand, IFNy-stimulation induced recruitment of ubiquitin to the PVM which could also recruit its adaptor proteins p62 and subsequently LC3B and GABARAP, which together can lead to endo-lysosomal fusion with the PVM and help in elimination of the parasites.

b) In MEFs, GKS IRGs (IRGB6 in this study) are recruited to the PVM upon IFNy-stimulation. We observed a decrease in IRG loading in Traf6/- MEFs suggesting that TRAF6 might act upstream of IRG loading. However, these IRGs also recruit other E3 ubiquitin ligases which can cause PVM ubiquitination which in turn could recruit p62. GRA15 by binding to TRAF6 accelerates the process and recruits more p62, LC3B and IRGs. This subsequently leads to enhanced recruitment of GBPs and damage to the PVM which ultimately kills the parasites. 


\section{References}

Alaganan A, Fentress SJ, Tang K, Wang Q \& Sibley LD (2014) Toxoplasma GRA7 effector increases turnover of immunity-related GTPases and contributes to acute virulence in the mouse. Proc. Natl. Acad. Sci. U. S. A. 111: 1126-1131

Bando H, Sakaguchi N, Lee Y, Pradipta A, Ma JS, Tanaka S, Lai D-H, Liu J, Lun Z-R, Nishikawa Y, Sasai M \& Yamamoto M (2018) Toxoplasma Effector TgIST Targets Host IDO1 to Antagonize the IFN-Y-Induced Anti-parasitic Response in Human Cells. Front. Immunol. 9: 1-14 Available at: https://www.frontiersin.org/article/10.3389/fimmu.2018.02073/full

Behnke MS, Khan A, Lauron EJ, Jimah JR, Wang Q, Tolia NH \& Sibley LD (2015) Rhoptry Proteins ROP5 and ROP18 Are Major Murine Virulence Factors in Genetically Divergent South American Strains of Toxoplasma gondii. PLoS Genet. 11: 1-22

Boyle JP, Saeij JPJ \& Boothroyd JC (2007) Toxoplasma gondii: Inconsistent dissemination patterns following oral infection in mice. Exp. Parasitol. 116: 302-305

Braun L, Brenier-Pinchart M-P, Yogavel M, Curt-Varesano A, Curt-Bertini R-L, Hussain T, Kieffer-Jaquinod S, Coute Y, Pelloux H, Tardieux I, Sharma A, Belrhali H, Bougdour A \& Hakimi M-A (2013) A Toxoplasma dense granule protein, GRA24, modulates the early immune response to infection by promoting a direct and sustained host p38 MAPK activation. J. Exp. Med. 210: 2071-2086 Available at: http://www.jem.org/lookup/doi/10.1084/jem.20130103

Broz P \& Dixit VM (2016) Inflammasomes: Mechanism of assembly, regulation and signalling. Nat. Rev. Immunol. 16: 407-420

Choi J, Park S, Biering SB, Selleck E, Liu CY, Zhang X, Fujita N, Saitoh T, Akira S, Yoshimori T, Sibley LD, Hwang S \& Virgin HW (2014) The Parasitophorous Vacuole Membrane of Toxoplasma gondii Is Targeted for Disruption by Ubiquitin-like Conjugation Systems of Autophagy. Immunity 40: 924-935 Available at: http://www.sciencedirect.com/science/article/pii/S1074761314001861

Clough B, Wright JD, Pereira PM, Hirst EM, Johnston AC, Henriques R \& Frickel EM (2016) K63-Linked Ubiquitination Targets Toxoplasma gondii for Endo-lysosomal Destruction in IFNy-Stimulated Human Cells. PLoS Pathog. 12:

Davies CC, Mak TW, Young LS \& Eliopoulos AG (2005) TRAF6 is required for TRAF2-dependent CD40 signal transduction in nonhemopoietic cells. Mol. Cell. Biol. 25: 9806-9819 Available at: https://www.ncbi.nlm.nih.gov/pubmed/16260598

Degrandi D, Kravets E, Konermann C, Beuter-Gunia C, Klumpers V, Lahme S, Wischmann E, Mausberg AK, Beer-Hammer S \& Pfeffer K (2013) Murine Guanylate Binding Protein 2 (mGBP2) controls Toxoplasma gondii replication. Proc. Natl. Acad. Sci. 110: 294-299 Available at: http://www.pnas.org/cgi/doi/10.1073/pnas.1205635110

Deng L, Wang C, Spencer E, Yang L, Braun A, You J, Slaughter C, Pickart C \& Chen ZJ (2000) Activation of the IkappaB kinase complex by TRAF6 requires a dimeric ubiquitin-conjugating enzyme complex and a unique polyubiquitin chain. Cell 103: 351-361

Donald RG \& Roos DS (1993) Stable molecular transformation of Toxoplasma gondii: a selectable dihydrofolate reductase-thymidylate synthase marker based on drug-resistance mutations in malaria. Proc. Natl. Acad. Sci. U. S. A. 90: 11703-11707

Dutta J, Fan Y, Gupta N, Fan G \& Gélinas C (2006) Current insights into the regulation of programmed cell death by NF-kB. Oncogene 25: 6800 Available at: https://doi.org/10.1038/sj.onc.1209938

Etheridge RD, Alaganan A, Tang K, Lou HJ, Turk BE \& Sibley LD (2014) file://C:/Users/debmi/Downloads/citations (18).nbibThe Toxoplasma Pseudokinase ROP5 Forms Complexes with ROP18 and ROP17 Kinases that Synergize to Control Acute Virulence in Mice. Cell Host Microbe 15: 537-550 Available at: https://doi.org/10.1016/j.chom.2014.04.002

Fisch D, Yakimovich A, Clough B, Wright J, Bunyan M, Howell M, Mercer J \& Frickel E (2019) Defining hostpathogen interactions employing an artificial intelligence workflow. Elife 8:

Fleckenstein MC, Reese ML, Konen-Waisman S, Boothroyd JC, Howard JC \& Steinfeldt T (2012) A Toxoplasma gondii pseudokinase inhibits host IRG resistance proteins. PLoS Biol. 10: e1001358

Foltz C, Napolitano A, Khan R, Clough B, Hirst EM \& Frickel E-M (2017) TRIM21 is critical for survival of Toxoplasma gondii infection and localises to GBP-positive parasite vacuoles. Sci. Rep. 7: 5209 Available at: https://doi.org/10.1038/s41598-017-05487-7 
Franco M, Panas MW, Marino ND, Lee MCW, Buchholz KR, Kelly FD, Bednarski JJ, Sleckman BP, Pourmand N \& Boothroyd JC (2016) A novel secreted protein, MYR1, is central to Toxoplasma's manipulation of host cells. MBio 7: 1-16

García MG, Alaniz L, Lopes EC, Blanco G, Hajos SE \& Alvarez E (2005) Inhibition of NF-kB activity by BAY 11-7082 increases apoptosis in multidrug resistant leukemic T-cell lines. Leuk. Res. 29: 1425-1434 Available at: https://www.sciencedirect.com/science/article/pii/S014521260500192X?via\%3Dihub [Accessed November 6, 2018]

Gay G, Braun L, Brenier-Pinchart M-P, Vollaire J, Josserand V, Bertini R-L, Varesano A, Touquet B, De Bock P-J, Coute Y, Tardieux I, Bougdour A \& Hakimi M-A (2016) Toxoplasma gondii TgIST co-opts host chromatin repressors dampening STAT1-dependent gene regulation and IFN- $\gamma-$ mediated host defenses. J. Exp. Med. 213: 1779-1798 Available at: http://www.jem.org/lookup/doi/10.1084/jem.20160340

Hakimi M, Olias P \& David L (2017) crossm Toxoplasma Effectors Targeting Host. 30: 615-645

Haldar AK, Foltz C, Finethy R, Piro AS, Feeley EM, Pilla-Moffett DM, Komatsu M, Frickel E-M \& Coers J (2015) Ubiquitin systems mark pathogen-containing vacuoles as targets for host defense by guanylate binding proteins. Proc. Natl. Acad. Sci. 112: E5628-E5637 Available at: http://www.pnas.org/lookup/doi/10.1073/pnas.1515966112

Haldar AK, Saka HA, Piro AS, Dunn JD, Henry SC, Taylor GA, Frickel EM, Valdivia RH \& Coers J (2013) IRG and GBP Host Resistance Factors Target Aberrant, "Non-self" Vacuoles Characterized by the Missing of "Self" IRGM Proteins. PLOS Pathog. 9: 1-16 Available at: https://doi.org/10.1371/journal.ppat.1003414

Hill D \& Dubey JP (2002) Toxoplasma gondii: transmission, diagnosis and prevention. Clin. Microbiol. Infect. 8: 634-640 Available at: https://onlinelibrary.wiley.com/doi/abs/10.1046/j.1469-0691.2002.00485.x

Howard JC, Hunn JP \& Steinfeldt T (2011) The IRG protein-based resistance mechanism in mice and its relation to virulence in Toxoplasma gondii. Curr. Opin. Microbiol. 14: 414-421 Available at: http://dx.doi.org/10.1016/j.mib.2011.07.002

Howe DK \& Sibley LD (1995) Toxoplasma gondii comprises three clonal lineages: correlation of parasite genotype with human disease. J. Infect. Dis. 172: 1561-1566

Hunn JP, Koenen-Waisman S, Papic N, Schroeder N, Pawlowski N, Lange R, Kaiser F, Zerrahn J, Martens S \& Howard JC (2008) Regulatory interactions between IRG resistance GTPases in the cellular response to Toxoplasma gondii. EMBO J. 27: 2495-2509

Hunter CA \& Sibley LD (2012) Modulation of innate immunity by Toxoplasma gondii virulence effectors. Nat. Rev. Microbiol. 10: 766-778 Available at: http://dx.doi.org/10.1038/nrmicro2858

Jensen KDC, Wang Y, Wojno EDT, Shastri AJ, Hu K, Cornel L, Boedec E, Ong YC, Chien YH, Hunter CA, Boothroyd JC \& Saeij JPJ (2011) Toxoplasma polymorphic effectors determine macrophage polarization and intestinal inflammation. Cell Host Microbe 9: 472-483 Available at:

http://dx.doi.org/10.1016/j.chom.2011.04.015

Johnston AC, Piro A, Clough B, Siew M, Virreira Winter S, Coers J \& Frickel EM (2016) Human GBP1 does not localize to pathogen vacuoles but restricts Toxoplasma gondii. Cell. Microbiol. 18: 1056-1064

Jones TC, Yeh S \& Hirsch JG (1972) The interaction between Toxoplasma gondii and mammalian cells. I. Mechanism of entry and intracellular fate of the parasite. J. Exp. Med. 136: 1157-1172 Available at: https://www.ncbi.nlm.nih.gov/pubmed/5082671

Khaminets A, Hunn JP, Konen-Waisman S, Zhao YO, Preukschat D, Coers J, Boyle JP, Ong Y-C, Boothroyd JC, Reichmann G \& Howard JC (2010) Coordinated loading of IRG resistance GTPases on to the Toxoplasma gondii parasitophorous vacuole. Cell. Microbiol. 12: 939-961

Kravets E, Degrandi D, Ma Q, Peulen T-O, Klumpers V, Felekyan S, Kuhnemuth R, Weidtkamp-Peters S, Seidel CA \& Pfeffer K (2016) Guanylate binding proteins directly attack Toxoplasma gondii via supramolecular complexes. Elife 5:

Krishnamurthy S, Konstantinou EK, Young LH, Gold DA \& Saeij JPJ (2017) The human immune response to Toxoplasma: Autophagy versus cell death. PLoS Pathog. 13: 1-6

Lamark T, Svenning S \& Johansen T (2017) Regulation of selective autophagy: the p62/SQSTM1 paradigm. Essays Biochem. 61: 609-624 Available at: http://essays.biochemistry.org/lookup/doi/10.1042/EBC20170035

Lee Y, Sasai M, Ma JS, Sakaguchi N, Ohshima J, Bando H, Saitoh T, Akira S \& Yamamoto M (2015) P62 Plays a Specific Role in Interferon-y-Induced Presentation of a Toxoplasma Vacuolar Antigen. Cell Rep. 13: 223-233

Liehl P, Zuzarte-Luis V \& Mota MM (2015) Unveiling the pathogen behind the vacuole. Nat. Rev. Microbiol. 13: 589-598 Available at: http://dx.doi.org/10.1038/nrmicro3504 
bioRxiv preprint doi: https://doi.org/10.1101/2020.02.24.963496; this version posted February 26, 2020. The copyright holder for this preprint (which was not certified by peer review) is the author/funder, who has granted bioRxiv a license to display the preprint in perpetuity. It is made available under aCC-BY-NC-ND 4.0 International license.

Man SM, Karki R \& Kanneganti TD (2017) Molecular mechanisms and functions of pyroptosis, inflammatory caspases and inflammasomes in infectious diseases. Immunol. Rev. 277: 61-75

Mordue DG \& Sibley LD (1997) Intracellular fate of vacuoles containing Toxoplasma gondii is determined at the time of formation and depends on the mechanism of entry. J. Immunol. 159: 4452 LP - 4459 Available at: http://www.jimmunol.org/content/159/9/4452.abstract

Nadipuram SM, Kim EW, Vashisht AA, Lin AH, Bell HN, Coppens I, Wohlschlegel JA \& Bradley PJ (2016) In Vivo Biotinylation of the Toxoplasma Parasitophorous Vacuole Reveals Novel Dense Granule Proteins Important for Parasite Growth and Pathogenesis. MBio 7: e00808-16 Available at: https://www.ncbi.nlm.nih.gov/pubmed/27486190

Naor A, Panas MW, Marino N, Coffey MJ, Tonkin CJ \& Boothroyd JC (2018) MYR1-Dependent Effectors Are the Major Drivers of a Host Cell's Early Response to Toxoplasma, Including Counteracting MYR1Independent Effects. MBio 9: Available at: http://mbio.asm.org/content/9/2/e02401-17.abstract

Nesvizhskii Al, Keller A, Kolker E \& Aebersold R (2003) A statistical model for identifying proteins by tandem mass spectrometry. Anal. Chem. 75: 4646-4658

Niedelman W, Gold DA, Rosowski EE, Sprokholt JK, Lim D, Arenas AF, Melo MB, Spooner E, Yaffe MB \& Saeij JPJ (2012) The rhoptry proteins ROP18 and ROP5 mediate Toxoplasma gondii evasion of the murine, but not the human, interferon-gamma response. PLoS Pathog. 8:

Niedelman W, Sprokholt JK, Clough B, Frickel EM \& Saeij JPJ (2013) Cell death of gamma interferonstimulated human fibroblasts upon Toxoplasma gondii infection induces early parasite egress and limits parasite replication. Infect. Immun. 81: 4341-4349

Olias P, Etheridge RD, Zhang Y, Holtzman MJ \& Sibley LD (2016) Toxoplasma Effector Recruits the Mi2/NuRD Complex to Repress STAT1 Transcription and Block IFN-y-Dependent Gene Expression. Cell Host Microbe 20: 72-82 Available at: http://dx.doi.org/10.1016/j.chom.2016.06.006

Pfefferkorn ER (1984) Interferon gamma blocks the growth of Toxoplasma gondii in human fibroblasts by inducing the host cells to degrade tryptophan. Proc. Natl. Acad. Sci. 81: 908 LP - 912 Available at: http://www.pnas.org/content/81/3/908.abstract

Pfefferkorn ER, Eckel M \& Rebhun S (1986) Interferon-y suppresses the growth of Toxoplasma gondii in human fibroblasts through starvation for tryptophan. Mol. Biochem. Parasitol. 20: 215-224 Available at: https://www.sciencedirect.com/science/article/pii/0166685186901015?via\%3Dihub [Accessed November $20,2018]$

Pleyer U, Schlüter D \& Mänz M (2014) Ocular Toxoplasmosis: Recent Aspects of Pathophysiology and Clinical Implications. Ophthalmic Res. 52: 116-123 Available at: https://www.karger.com/DOI/10.1159/000363141

Qin A, Lai D-H, Liu Q, Huang W, Wu Y-P, Chen X, Yan S, Xia H, Hide G, Lun Z-R, Ayala FJ \& Xiang AP (2017) Guanylate-binding protein 1 (GBP1) contributes to the immunity of human mesenchymal stromal cells against Toxoplasma gondii. Proc. Natl. Acad. Sci. 114: 1365-1370 Available at: http://www.pnas.org/lookup/doi/10.1073/pnas.1619665114

Rosowski EE, Lu D, Julien L, Rodda L, Gaiser RA, Jensen KDC \& Saeij JPJ (2011) Strain-specific activation of the NF-KB pathway by GRA15, a novel Toxoplasma gondii dense granule protein. J. Exp. Med. 208: 195212 Available at: http://www.jem.org/lookup/doi/10.1084/jem.20100717

Saeij JP \& Frickel EM (2017) Exposing Toxoplasma gondii hiding inside the vacuole: a role for GBPs, autophagy and host cell death. Curr. Opin. Microbiol. 40: 72-80 Available at: http://dx.doi.org/10.1016/j.mib.2017.10.021

Saeij JPJ, Boyle JP \& Boothroyd JC (2005) Differences among the three major strains of Toxoplasma gondii and their specific interactions with the infected host. Trends Parasitol. 21: 476-481

Sangaré LO, Yang N, Konstantinou EK, Lu D, Mukhopadhyay D, Young LH \& Saeij JPJ (2019) \&lt;em\&gt;Toxoplasma\&lt;/em\&gt; GRA15 Activates the NF-kB Pathway through Interactions with TNF Receptor-Associated Factors. MBio 10: e00808-19 Available at: http://mbio.asm.org/content/10/4/e0080819.abstract

Sasai M, Sakaguchi N, Ma JS, Nakamura S, Kawabata T, Bando H, Lee Y, Saitoh T, Akira S, Iwasaki A, Standley DM, Yoshimori T \& Yamamoto M (2017) Essential role for GABARAP autophagy proteins in interferon-inducible GTPase-mediated host defense. Nat. Immunol. 18: 899 Available at: https://doi.org/10.1038/ni.3767

Selleck EM, Fentress SJ, Beatty WL, Degrandi D, Pfeffer K, Virgin IV HW, MacMicking JD \& Sibley LD (2013) Guanylate-binding Protein 1 (Gbp1) Contributes to Cell-autonomous Immunity against Toxoplasma gondii. PLOS Pathog. 9: e1003320 Available at: https://doi.org/10.1371/journal.ppat.1003320

Selleck EM, Orchard RC, Lassen KG, Beatty WL, Xavier RJ, Levine B, Virgin HW \& David Sibley L (2015) A 
noncanonical autophagy pathway restricts toxoplasma gondii growth in a strain-specific manner in IFN- $\gamma^{-}$ activated human cells. MBio 6:

Shen B, Brown KM, Lee TD \& Sibley LD (2014) Efficient gene disruption in diverse strains of Toxoplasma gondii using CRISPR/CAS9. MBio 5: e01114-14

Steinfeldt T, Konen-Waisman S, Tong L, Pawlowski N, Lamkemeyer T, Sibley LD, Hunn JP \& Howard JC (2010) Phosphorylation of mouse immunity-related GTPase (IRG) resistance proteins is an evasion strategy for virulent Toxoplasma gondii. PLoS Biol. 8: e1000576

Strickson S, Campbell DG, Emmerich CH, Knebel A, Plater L, Ritorto MS, Shpiro N \& Cohen P (2013) The anti-inflammatory drug BAY 11-7082 suppresses the MyD88-dependent signalling network by targeting the ubiquitin system. Biochem. J. 451: 427-437

Swatek KN \& Komander D (2016) Ubiquitin modifications. Cell Res. 26: 399 Available at: https://doi.org/10.1038/cr.2016.39

Virreira Winter S, Niedelman W, Jensen KD, Rosowski EE, Julien L, Spooner E, Caradonna K, Burleigh BA, Saeij JPJ, Ploegh HL \& Frickel EM (2011) Determinants of GBP recruitment to Toxoplasma gondii vacuoles and the parasitic factors that control it. PLoS One 6:

Wang P, Li S, Zhao Y, Zhang B, Li Y, Liu S, Du H, Cao L, Ou M, Ye X, Li P, Gao X, Wang P, Jing C, Shao F, Yang G \& You F (2019a) The GRA15 protein from Toxoplasma gondii enhances host defense responses by activating the interferon stimulator STING. J. Biol. Chem.

Wang Y, Cirelli KM, Barros PDC, Sangaré LO, Butty V, Hassan MA, Pesavento P, Mete A \& Saeij JPJ (2019b) Three Toxoplasma gondii Dense Granule Proteins Are Required for Induction of Lewis Rat Macrophage Pyroptosis. MBio 10file:///: e02388-18 Available at: http://mbio.asm.org/content/10/1/e02388-18.abstract

Yang N, Farrell A, Niedelman W, Melo M, Lu D, Julien L, Marth GT, Gubbels MJ \& Saeij JPJ (2013) Genetic basis for phenotypic differences between different Toxoplasma gondii type I strains. BMC Genomics 14:

Zhao YO, Khaminets A, Hunn JP \& Howard JC (2009) Disruption of the Toxoplasma gondii parasitophorous vacuole by IFNy-inducible immunity-related GTPases (IRG proteins) triggers necrotic cell death. PLoS Pathog. 5: 
Figure 1

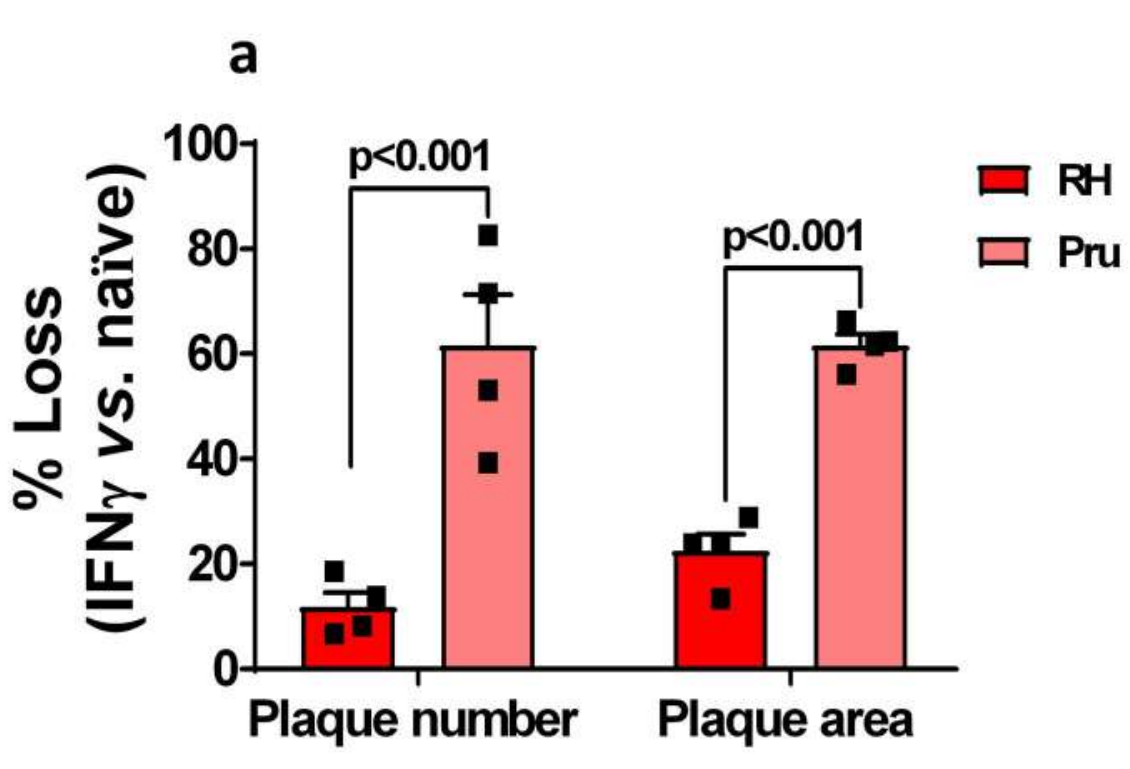

b

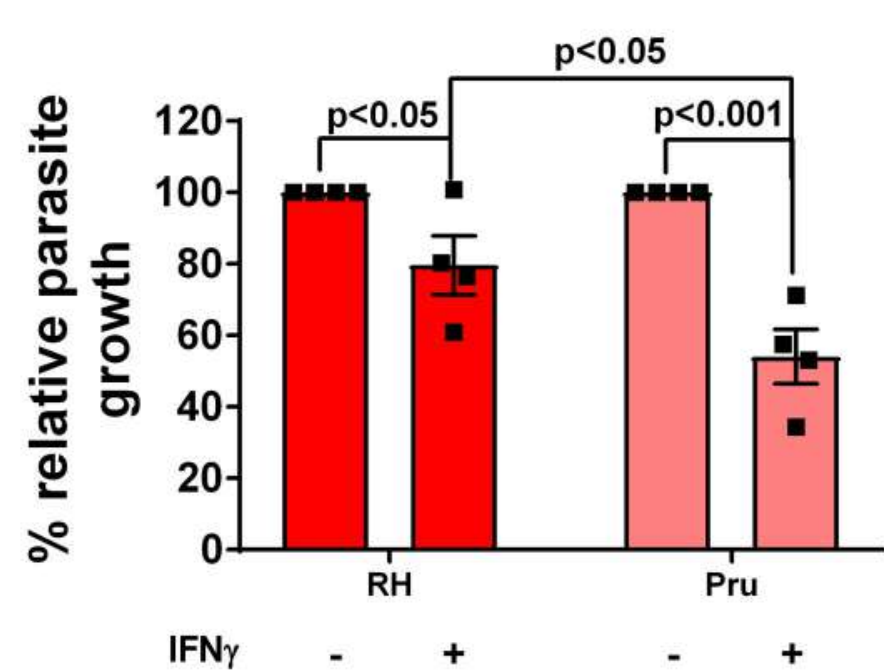

c

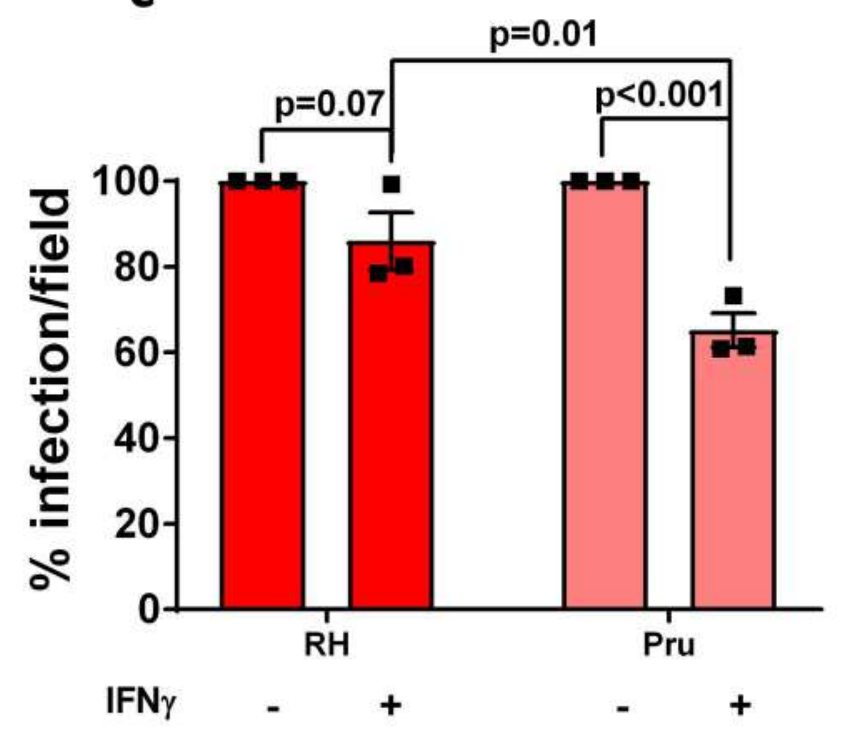

d

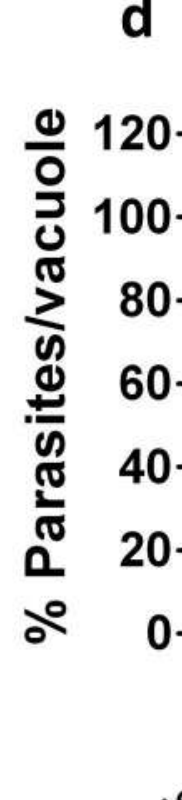

口 >4 parasites/vacuole 口 4 parasites/vacuole ロ 2 parasites/vacuole 口 1 parasites/vacuole
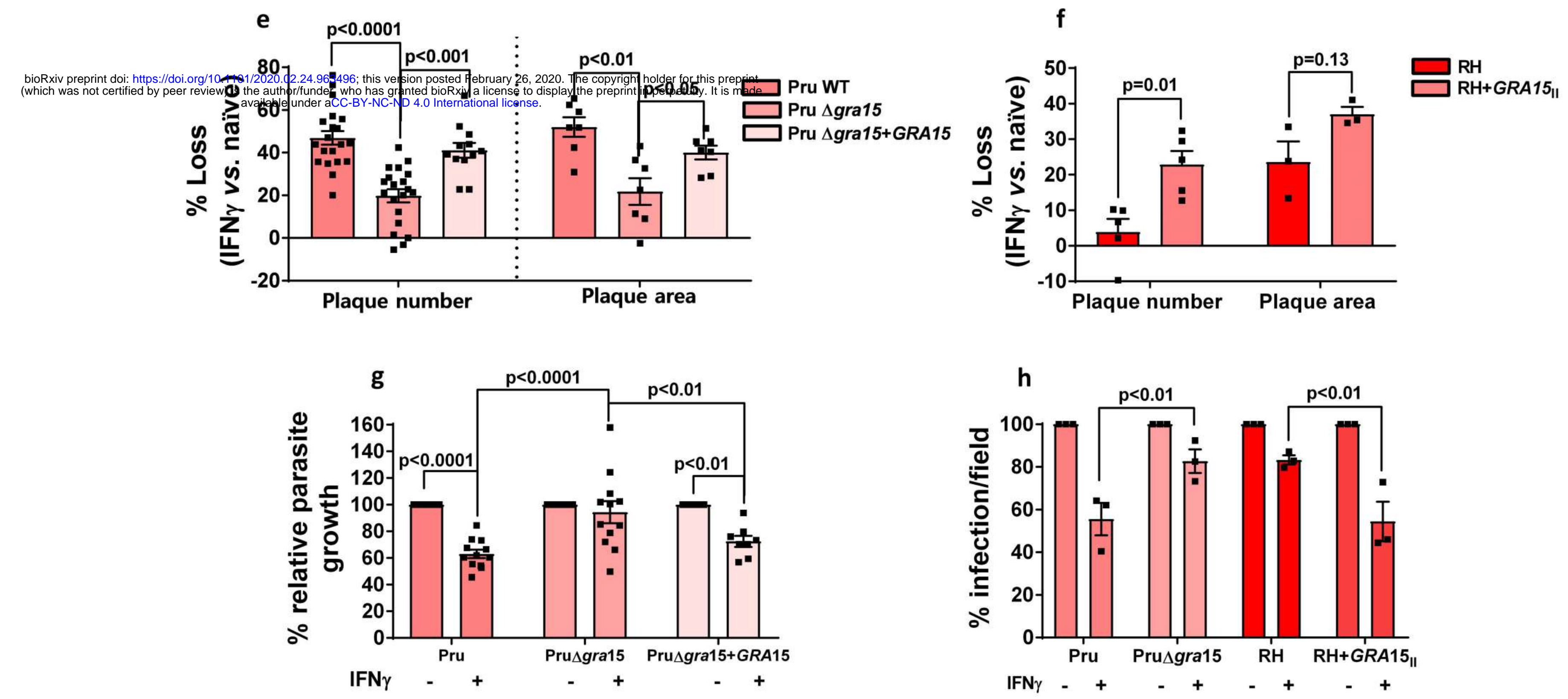
Figure 2
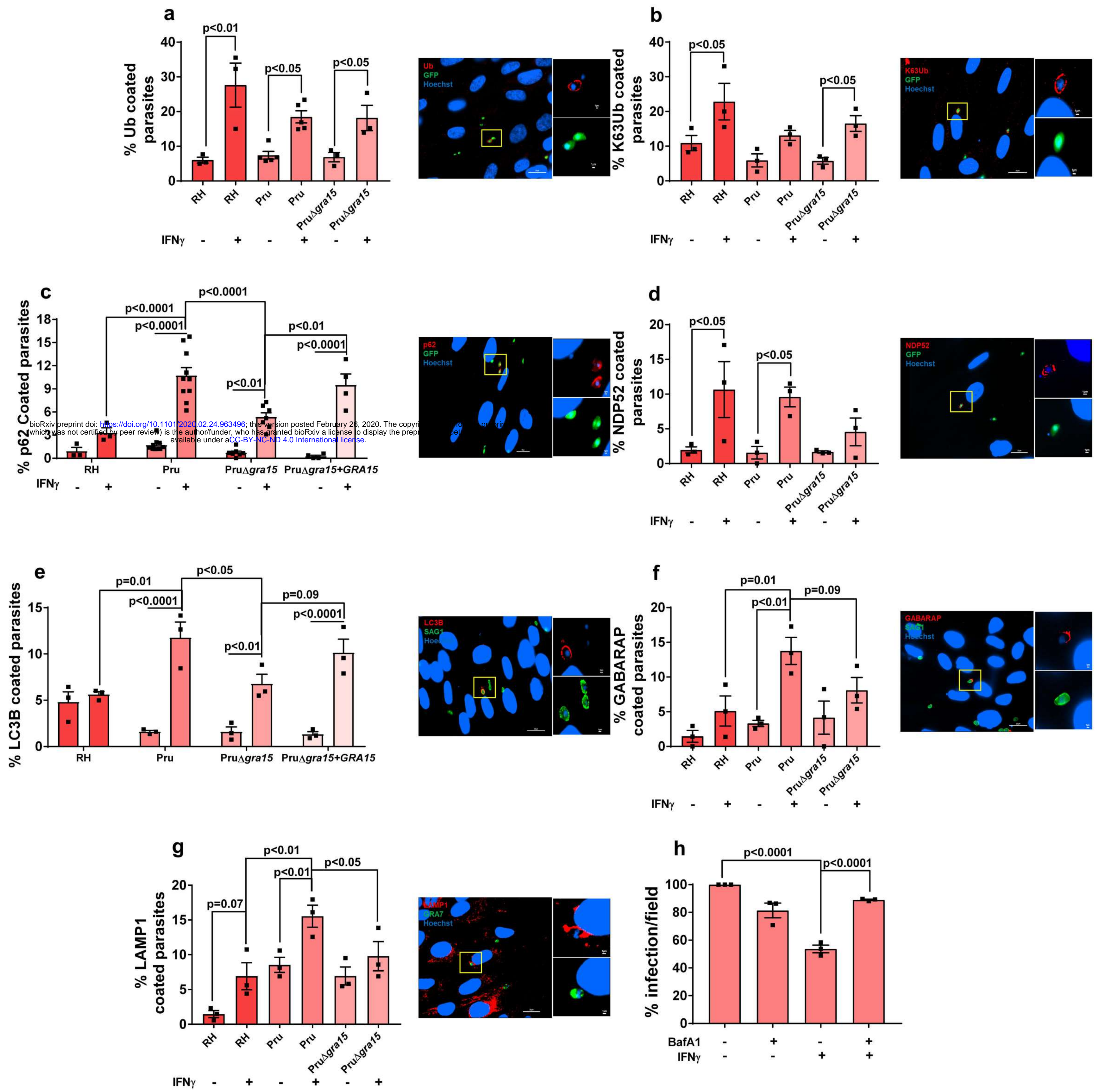
Figure 3

a

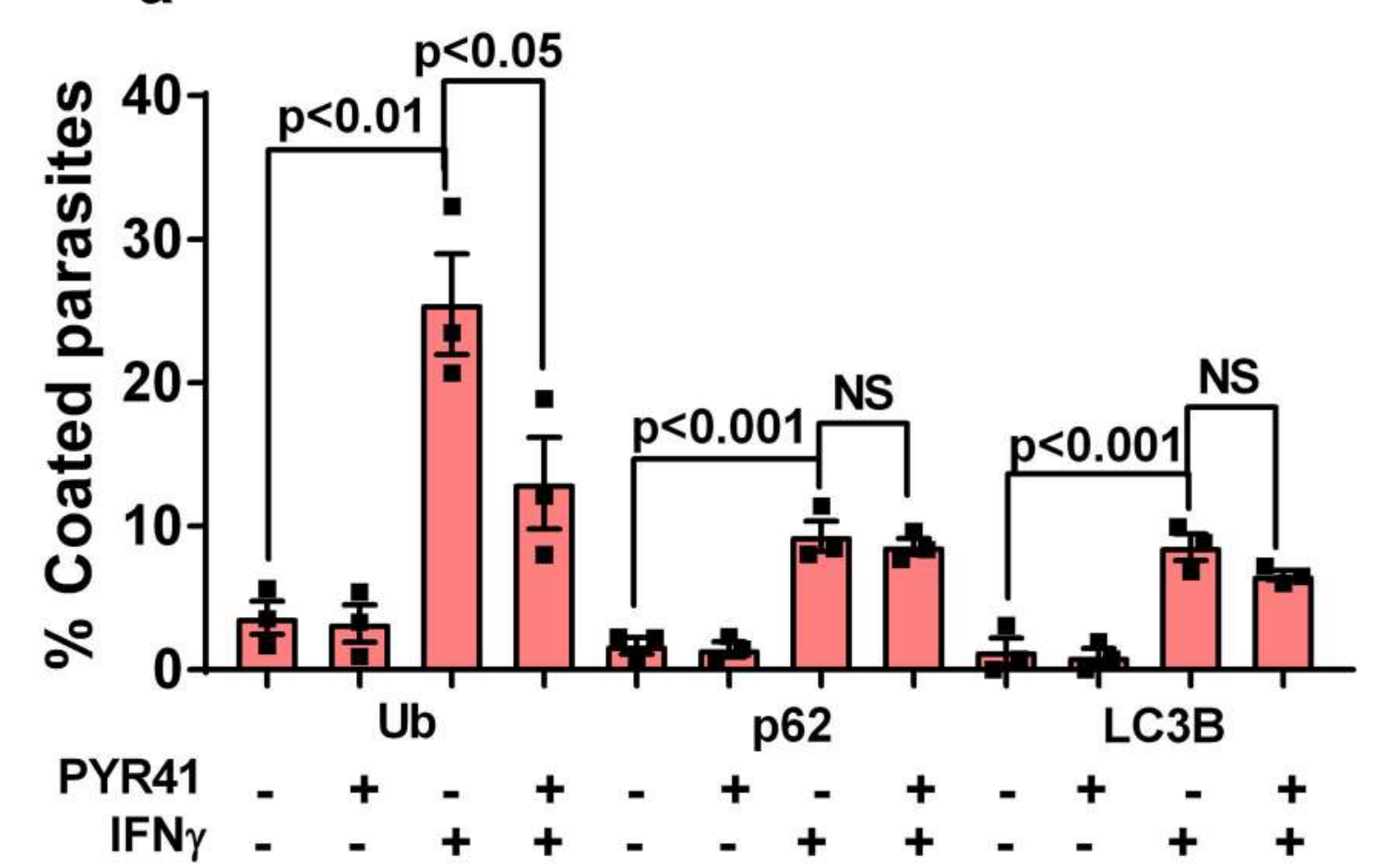

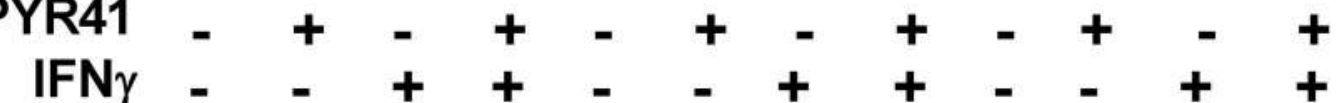
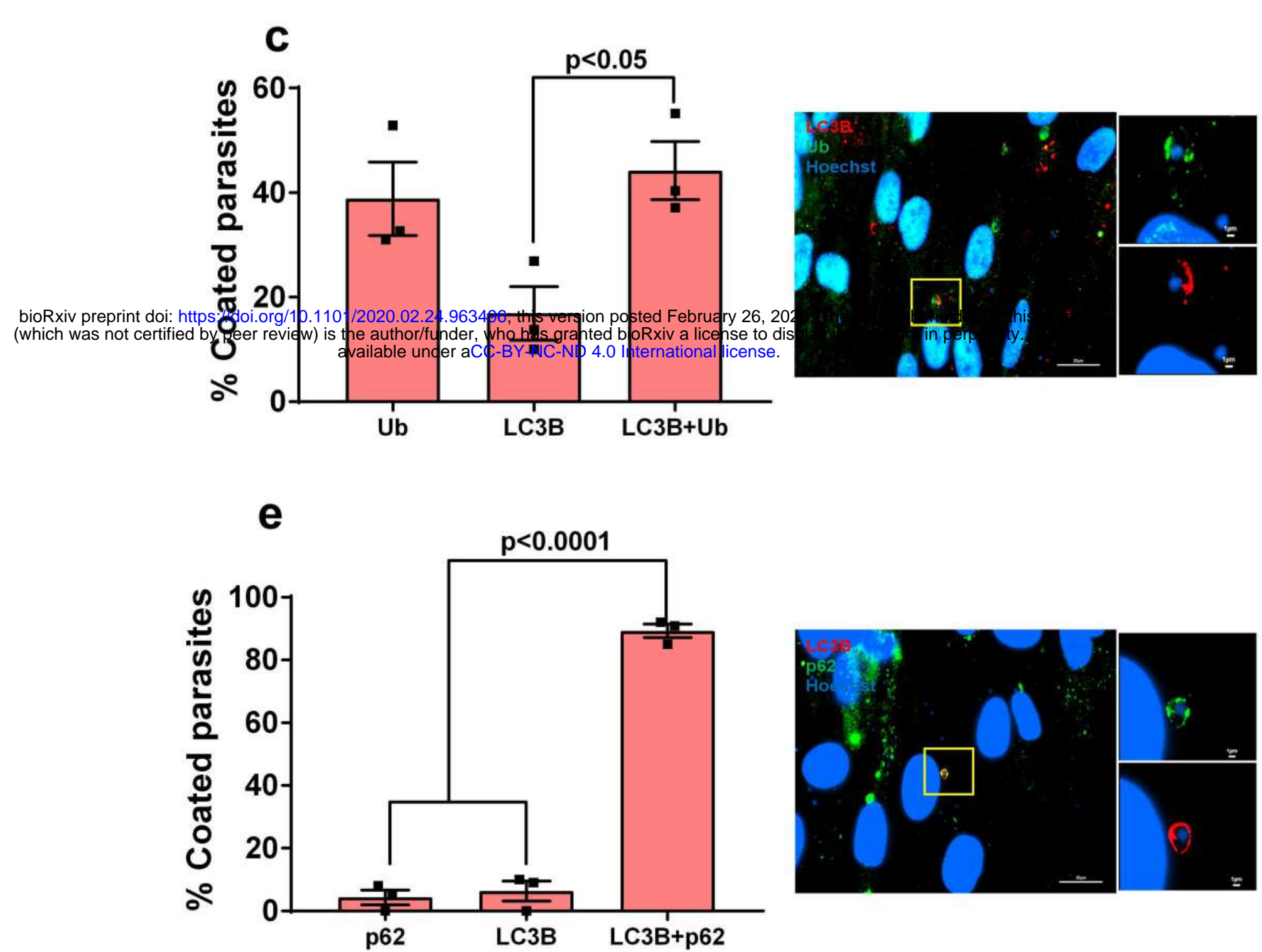

b
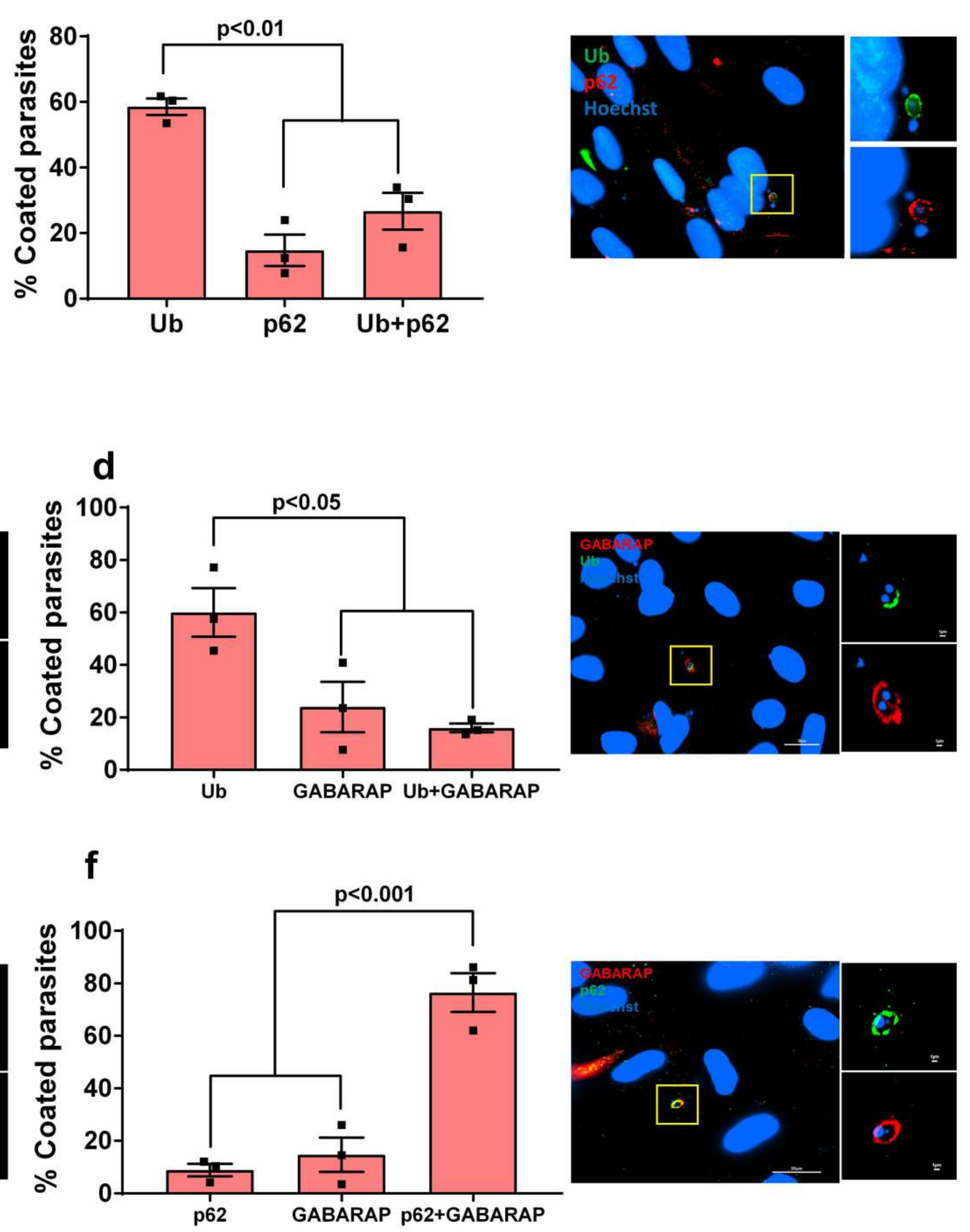

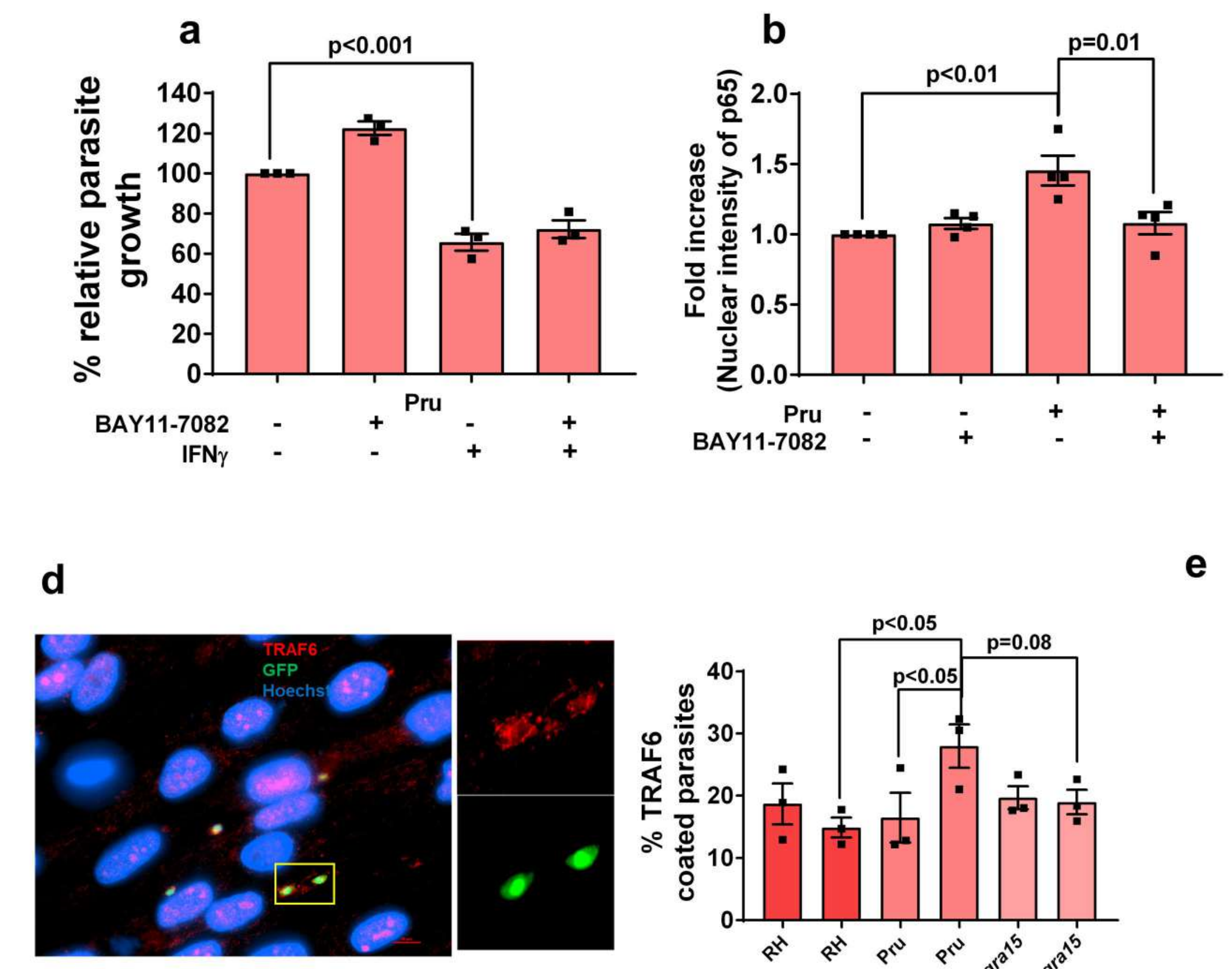

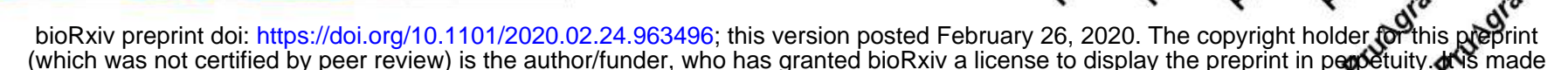
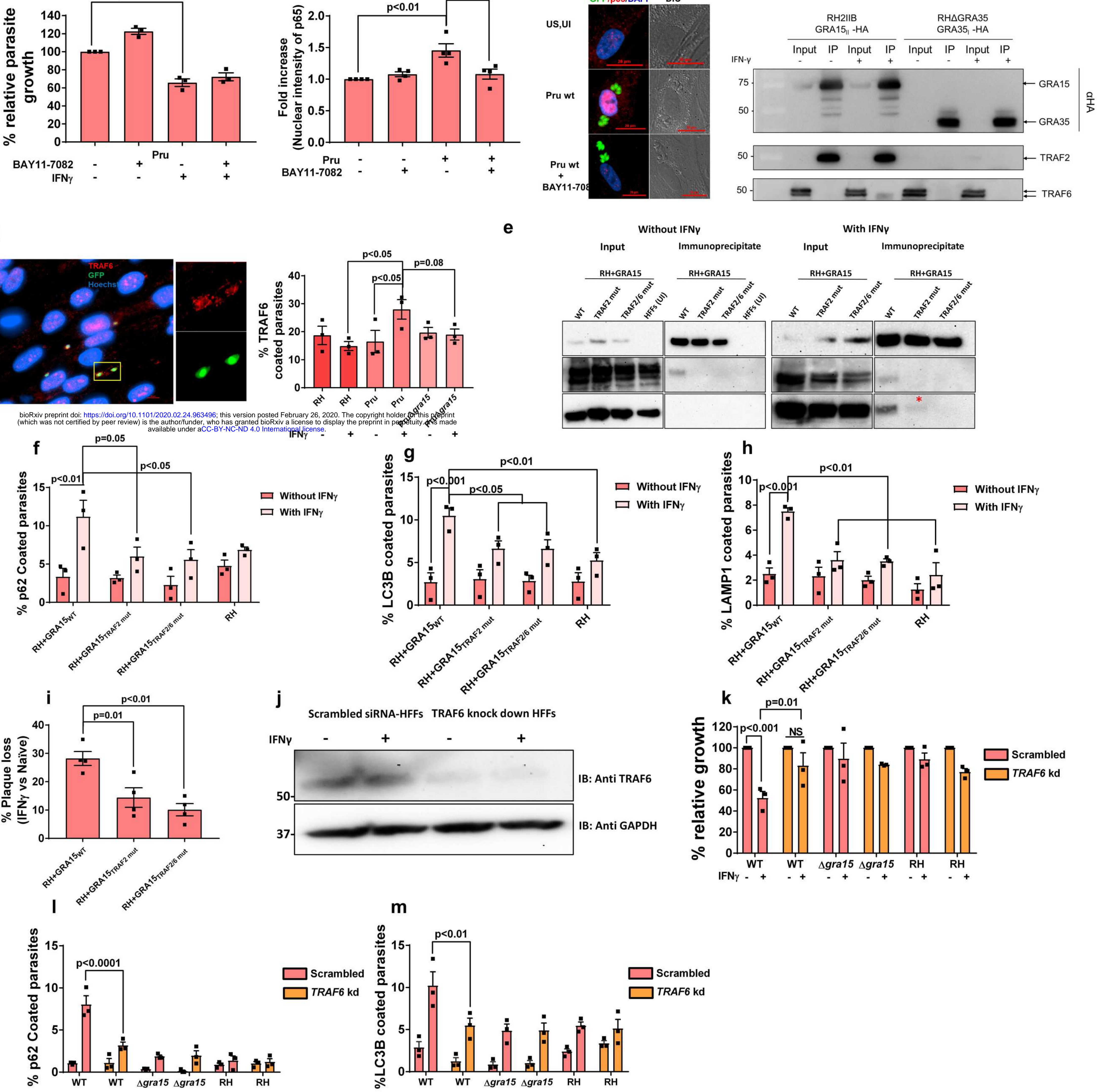

Figure 4 
Figure 5
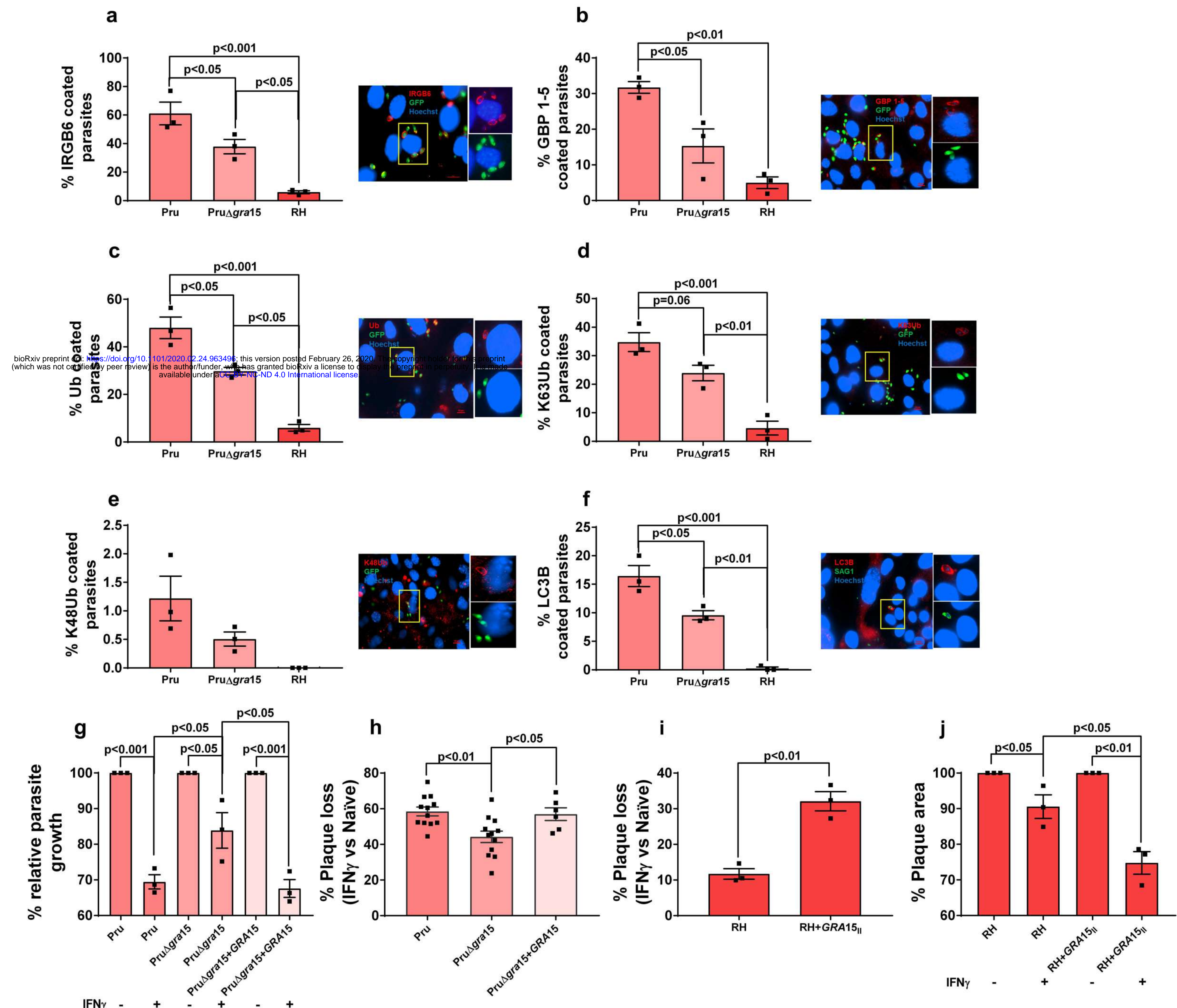
RH+GRA15 | RH+GRA45 | RH+GRA15 | $R H+G R A 45$, Input Immunoprecipitate

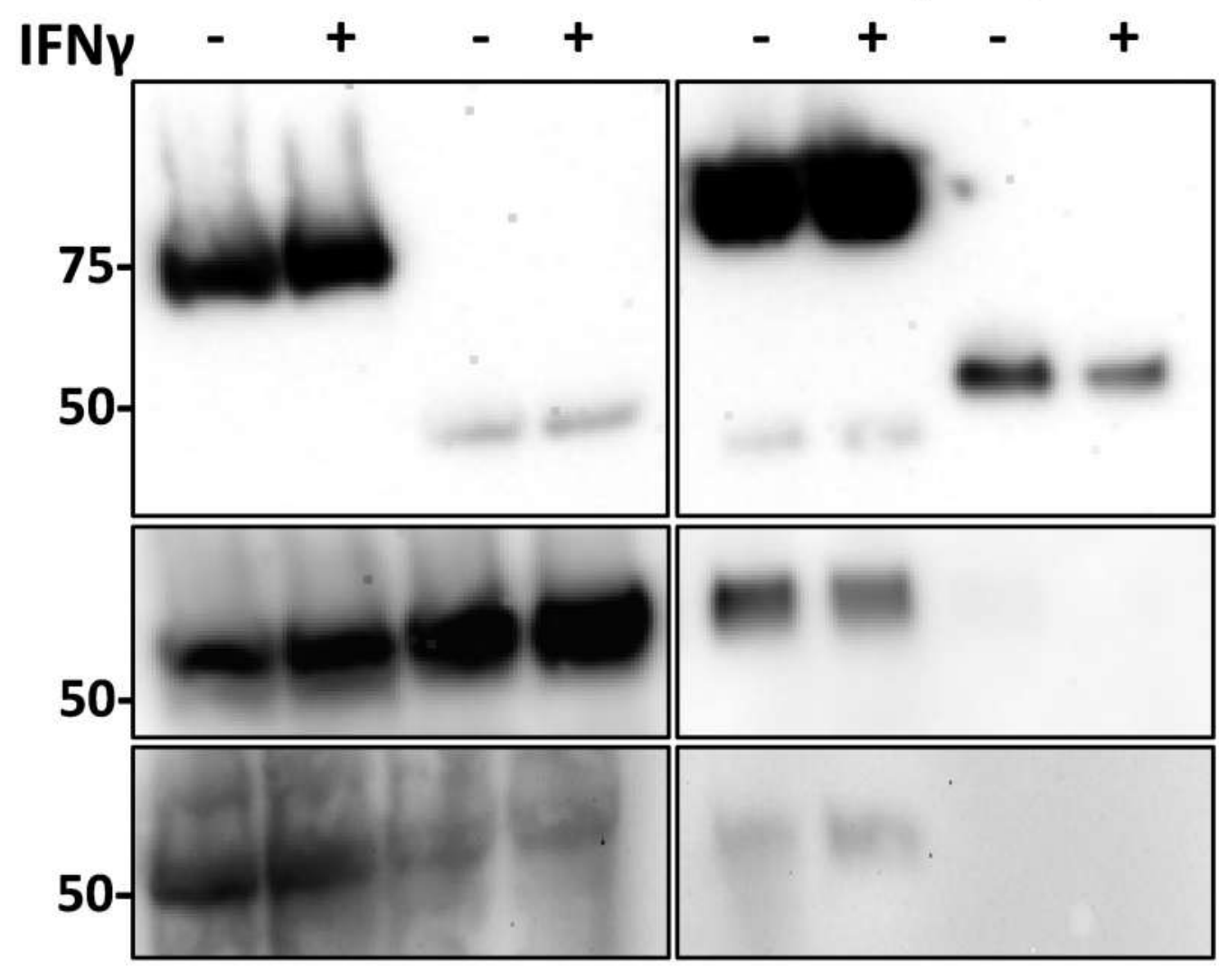

B: Anti HA
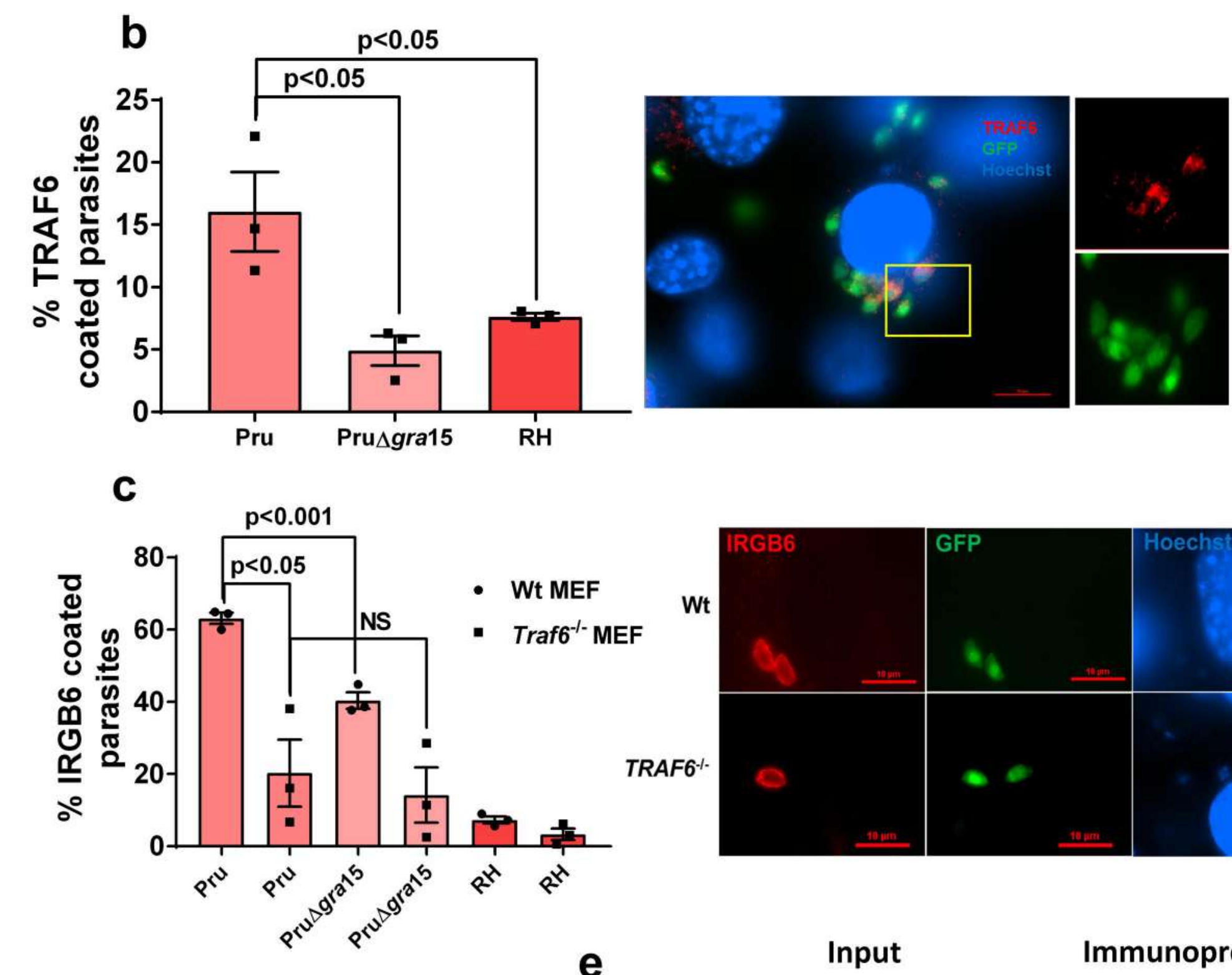

IB: Anti TRAF6

IB: Anti TRAF2

e

I.

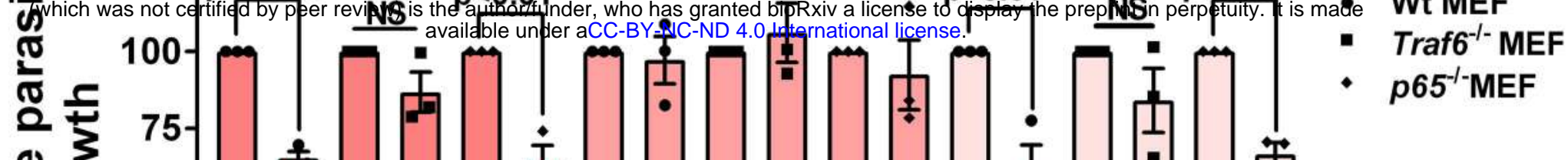

$\stackrel{2}{0} 7$

$\sum_{\text {¿ }}^{0}$ 잉 50

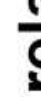

ㅇำ

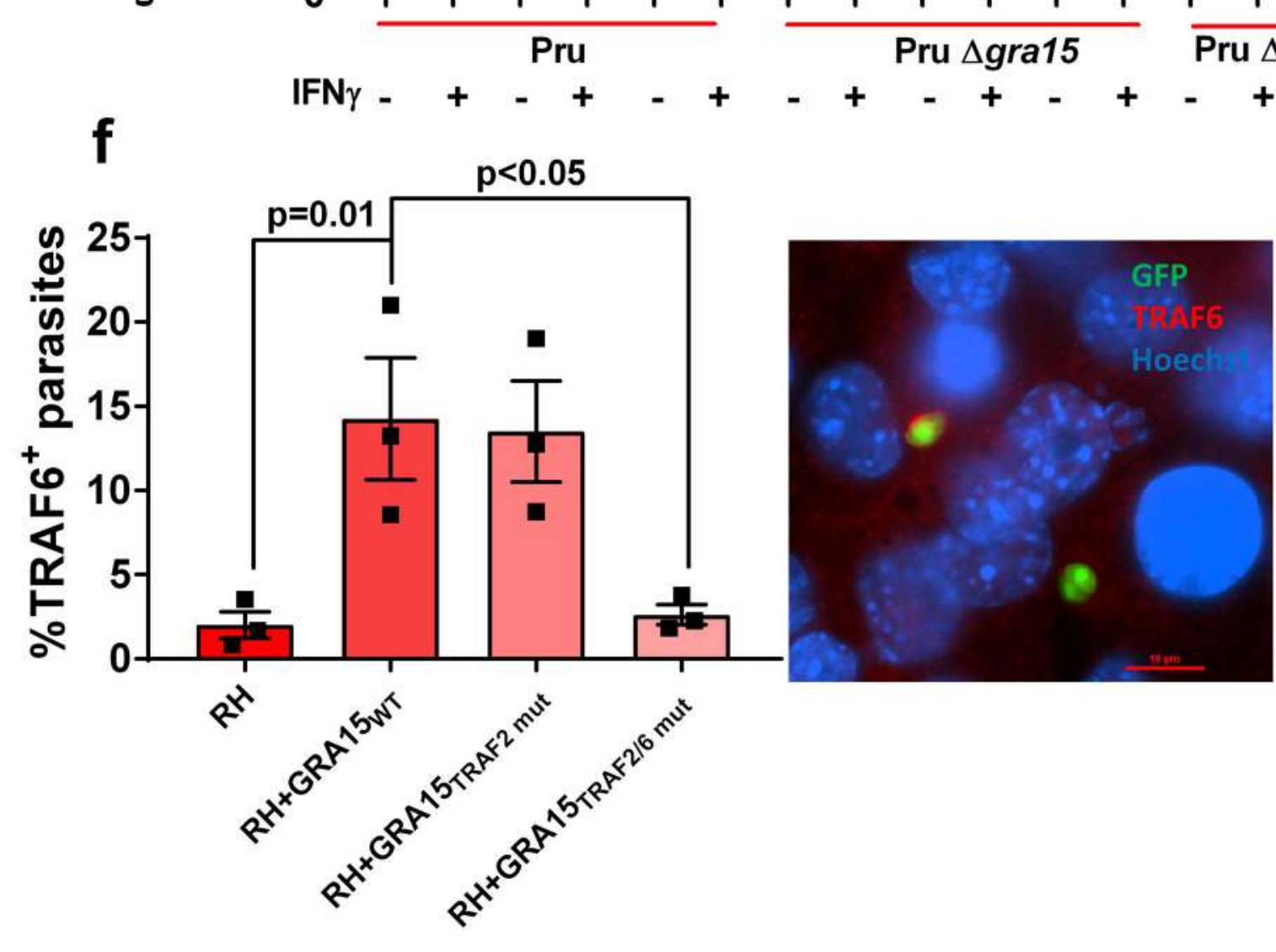

\section{$\triangle$ gra15 + GRA15}
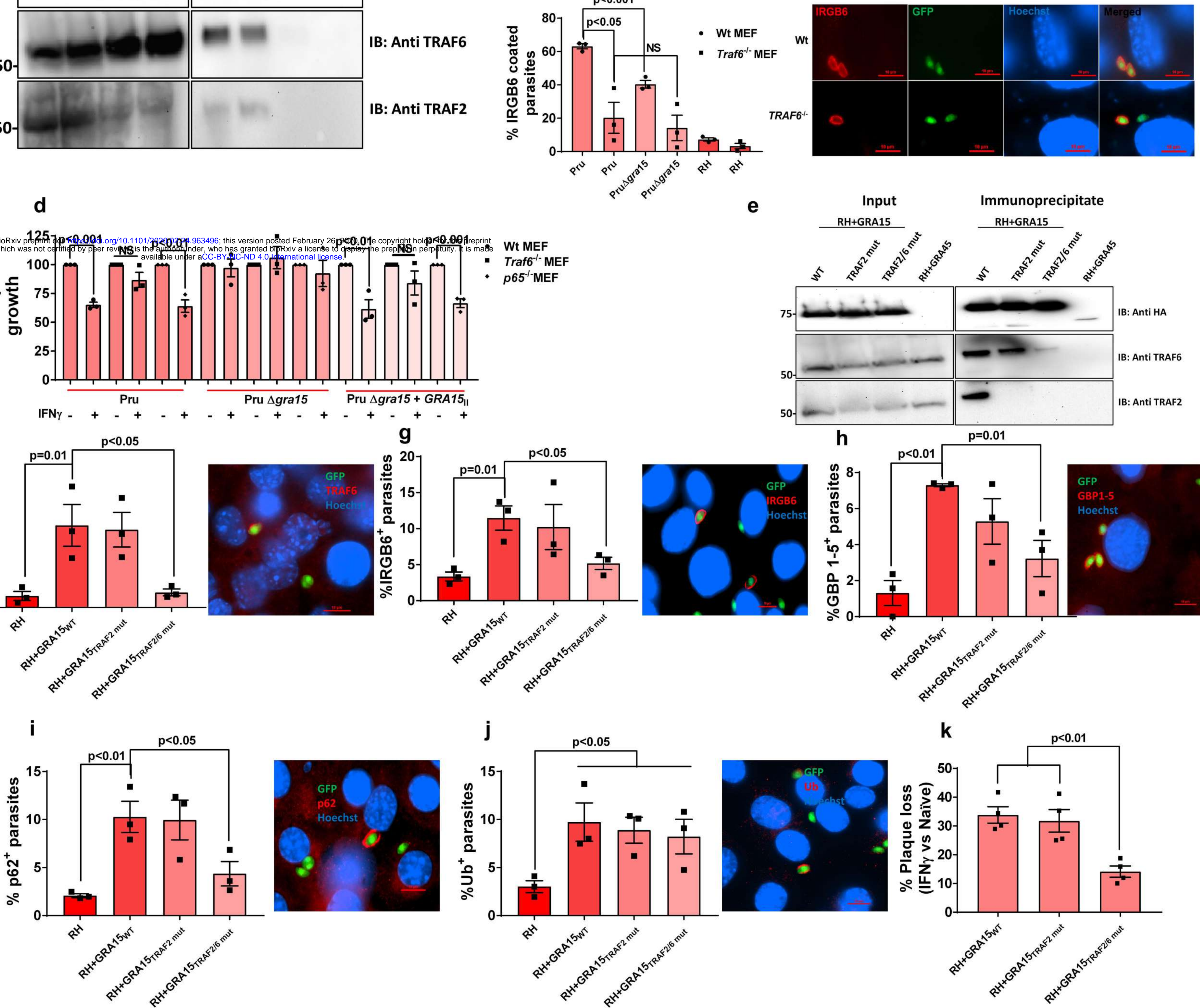
Figure 7

Human fibroblasts

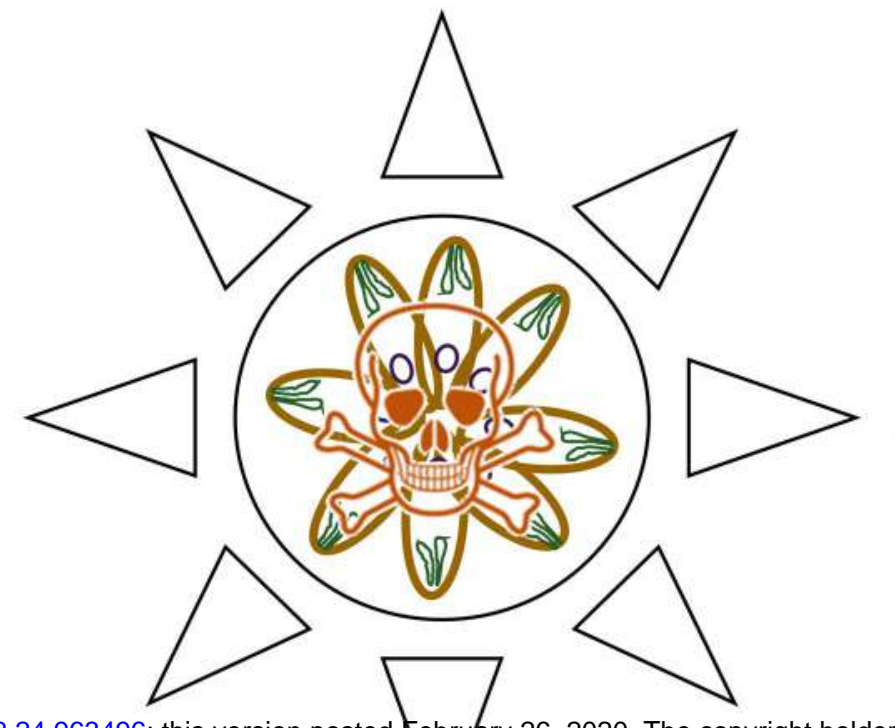

AMP1 coating) Endolysosomal damage

(BafA1 restorable)

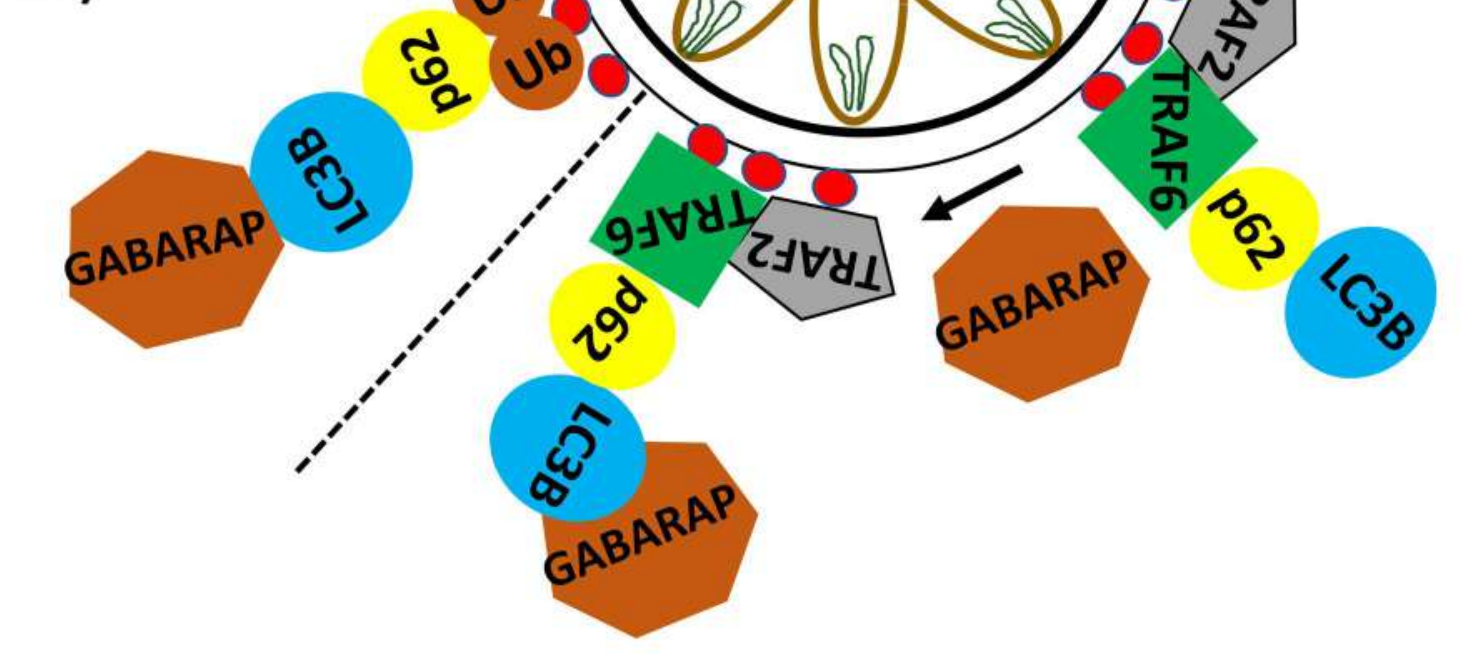

b

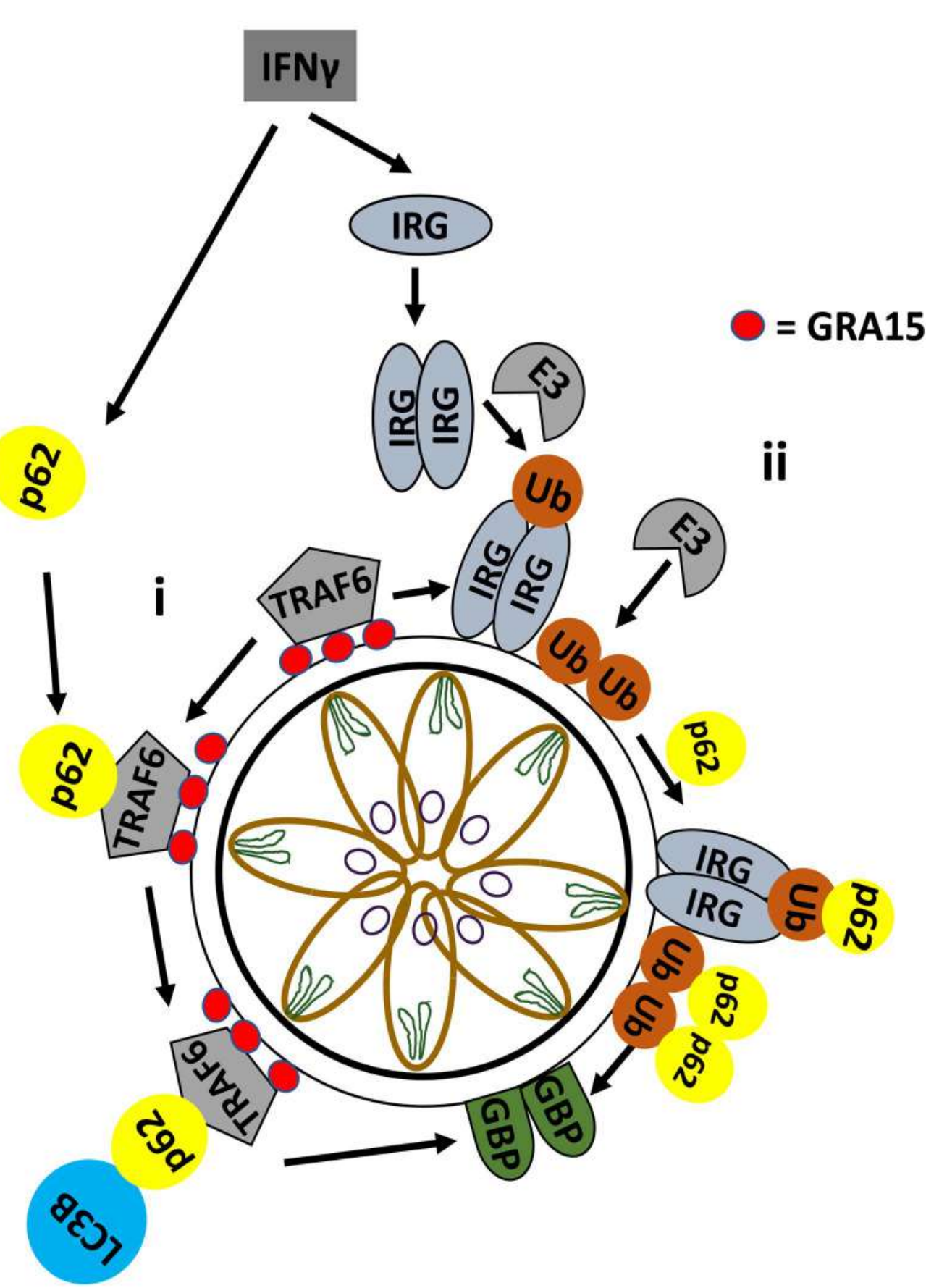

$\stackrel{\text { Rupture of }}{\longrightarrow}$ PVM

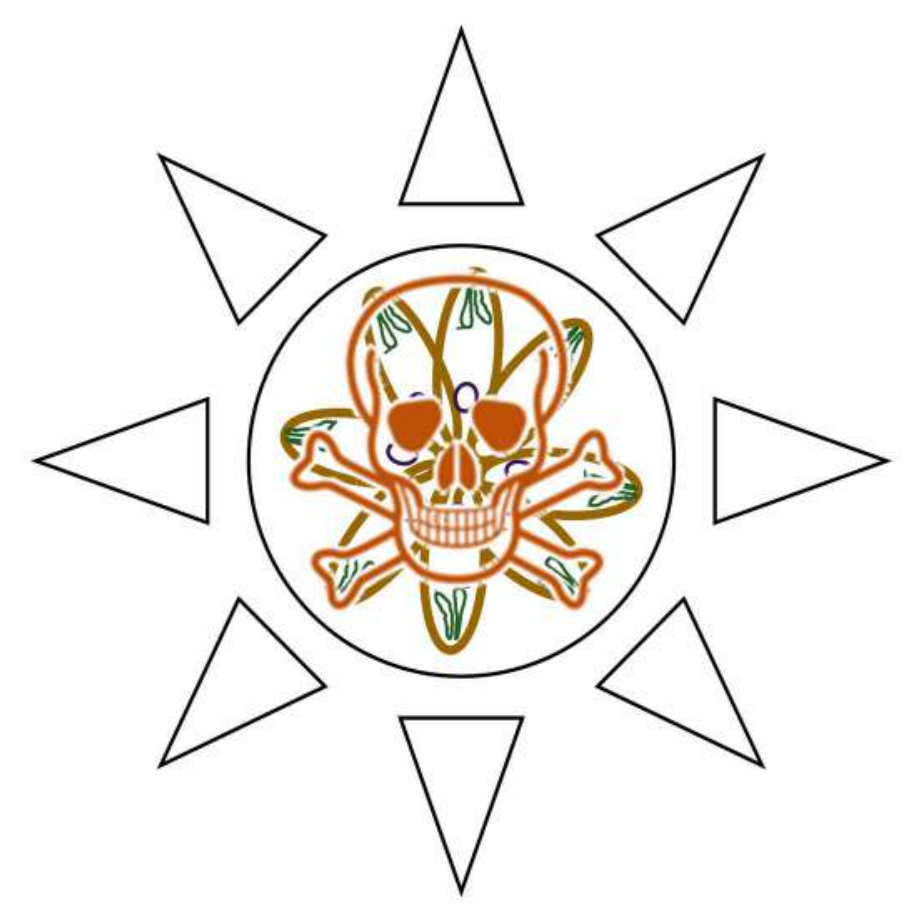

